\title{
GLOBAL ATTRACTOR FOR A NONLOCAL MODEL FOR BIOLOGICAL AGGREGATION*
}

\author{
CIPRIAN G. GAL ${ }^{\dagger}$
}

\begin{abstract}
We investigate the long term behavior in terms of global attractors, as time goes to infinity, of solutions to a continuum model for biological aggregations in which individuals experience long-range social attraction and short range dispersal. We consider the aggregation equation with both degenerate and non-degenerate diffusion in a bounded domain subject to various boundary conditions. In the degenerate case, we prove the existence of the global attractor and derive some optimal regularity results. Furthermore, in the non-degenerate case we give a complete structural characterization of the global attractor, and also discuss the convergence of any bounded solutions to steady states. In particular, under suitable assumptions on the parameters of the problem, we establish the convergence of the bounded solution $u(t)$ to a single steady state $u_{*}$, and the rate of convergence

$$
\left\|u(t)-u_{*}\right\|_{L^{p}(\Omega)} \sim(1+t)^{-\rho}, \text { as } t \rightarrow \infty,
$$

for any $p>1$, and some $\rho=\rho\left(u_{*}, p\right) \in(0,1)$. Finally, the existence of an exponential attractor is also demonstrated for sufficiently smooth kernels in the case of non-degenerate diffusion. Our analysis extends and complements the analysis from [17] and many other fundamental works.
\end{abstract}

Key words. Global existence, gradient structure, global attractor, convergence to steady states, exponential attractor, chemotaxis, biological aggregation.

AMS subject classifications. 35K55, 35A01, 35A02.

\section{Introduction}

In this article, we are interested in the asymptotic behavior, as time goes to infinity, of general aggregation models with (non)degenerate diffusion of the form

$$
\partial_{t} u+\nabla \cdot(u \vec{V})=\Delta A(u), \text { in } \Omega \times(0, \infty),
$$

where $\vec{V}=\nabla \mathcal{K} * u$, and $\Omega$ is a bounded domain in $\mathbb{R}^{d}, d \geq 1$. The kernel $\mathcal{K}$ incorporates the sensing range and degradation for the particular population density $u$ under consideration, while the term on the right-hand side of (1.1) models the dispersal mechanism, such as local repulsion. We aim to investigate (1.1) with both no-flux boundary conditions and Dirichlet boundary conditions. More precisely, we wish to consider the situation in which the boundary $\partial \Omega$ of the domain $\Omega$ consists of two disjoint open subsets $\Gamma_{N} \neq \varnothing$ and $\Gamma_{D}$ (possibly empty), each $\bar{\Gamma}_{i} \Gamma_{i}(i \in\{D, N\})$ is a $\sigma$-null subset of $\partial \Omega$ and $\partial \Omega=\bar{\Gamma}_{N} \cup \bar{\Gamma}_{D}$; here, $\sigma$ denotes the restriction to $\partial \Omega$ of the $(d-1)$-dimensional Hausdorff measure which coincides with the Lebesgue surface measure, if we assume that $\partial \Omega$ is at least Lipschitz. Thus, we consider the following boundary conditions for (1.1):

$$
\begin{cases}(\nabla A(u)-u \vec{V}) \cdot \vec{n}=0, & \text { on } \Gamma_{N} \times(0, \infty), \\ u=0, & \text { on } \Gamma_{D} \times(0, \infty),\end{cases}
$$

and initial condition

$$
u_{\mid t=0}=u_{0} \text { in } \Omega \text {. }
$$

${ }^{*}$ Received: November 30, 2012; accepted (in revised form): April 27, 2013. Communicated by Martin Burger.

${ }^{\dagger}$ Department of Mathematics, Florida International University, Miami, FL 33199, USA (cgal@ fiu.edu). 
The physical motivation for taking boundary conditions as in (1.2) is clear. For equation (1.1), the homogeneous boundary condition on $\Gamma_{D}$ may be interpreted as if the species suffers extinction if say the patch $\Gamma_{D} \subset \partial \Omega$ where the individuals live is toxic. The no-flux boundary condition in (1.2) says that nothing can cross the boundary $\Gamma_{N}$. Dirichlet boundary conditions for the population density $u$ can also arise for reaction-diffusion systems in the modelling of competition between two population species whose interaction occurs mainly in a region where their habitats overlap. This gives rise to Dirichlet boundary conditions for either species on the whole $\partial \Omega$ or only on a part $\Gamma_{D}$ of $\partial \Omega$ (see [44, 49] for the biological literature; cf. also [13, 42], for some mathematical results). We aim to give some results which allow one to deduce the $L^{p}-L^{\infty}$, and then the $L^{\infty}-C^{\alpha}(\bar{\Omega})$ smoothing properties for solutions of (1.1)-(1.3) assuming that some sort of energy estimate is a priori known in $L^{p}$-norm for some finite $p$. The main tool will be an iterative argument following a well-known Alikakos-Moser technique combined with a suitable form of Gronwall's inequality and a refined ODE argument. Our estimates are much stronger than those obtained in $[12,17]$ since our constants are uniform with respect to time and the initial data. It is well-known that the above smoothing properties become essential tools in attractor theory, where they can be used to establish the existence of an absorbing set in the $C^{\alpha}(\bar{\Omega})$-norm if this property can be deduced easily in the $L^{p}$-norm for some finite p. Recall that a subset $\mathcal{V} \subset \mathcal{H}$, where $\mathcal{H}$ is a topological space endowed with a given metric, is called absorbing if the orbits corresponding to bounded sets $B$ of initial data enter into $\mathcal{V}$ after a certain time (which may depend on the set $B$ ) and will stay there forever. If the space $\mathcal{V}$ is further compactly embedded in $\mathcal{H}$, then the existence of the global attractor for a system like (1.1)-(1.3) follows from standard abstract results (see $[46,51]$ ). The global attractor for (1.1)-(1.3) encodes all the information about the the long-time behavior of solutions to (1.1)-(1.3) departing from bounded sets of initial data. Our aim is to establish such a result for our system. We will consider diffusions like $A(y) \sim y^{m}$, for some $m>m_{*} \geq 1$ (the subcritical case). This scenario is in complete agreement with biological observation, that only in the case of degenerate diffusion there are solutions of (1.1)-(1.3) which have compact support, steep edges, and a constant internal population density (cf. [53]). We shall also emphasize the role of boundary conditions (see (1.2)), and how they affect various dissipative estimates. In fact, when $\sigma\left(\Gamma_{D}\right)=0$, and $m_{*}=1+1 / \gamma-2 / d$, for any $1 \leq \gamma \leq d / 2$, we extend the results in [17] to show the existence of a global attractor, bounded in $C^{\alpha}(\bar{\Omega})$, for some $\alpha \in(0,1)$, as long as $m>m_{*}$. In the critical case $m=m_{*}$, the same result is valid provided that for $\int_{\Omega} u_{0} d x=\widetilde{M}$ (see (2.1) below), we have $\widetilde{M}<M_{c}$, where $M_{c}$ is the critical mass estimated in [17, Theorem 7 and Proposition 3]. However, when $\sigma\left(\Gamma_{D}\right)>0$ it appears that we cannot recover the critical exponent $m_{*}$ as above. We can only show the existence of the global attractor, bounded in $C^{\alpha}(\bar{\Omega})$, provided that $m>m_{*}=2$ in this case. This appears to be due to loss of the conservation of mass property in the associated non-degenerate problem (i.e., if $A^{\prime}(y) \geq \varepsilon>0, \forall y \geq 0$ ) which becomes crucial in obtaining uniform a priori estimates for the degenerate problem (1.1)-(1.3). Finally, as in [17] we give optimal assumptions on the interaction kernel $\mathcal{K}$ which include important cases of interest, such as the Newtonian and Bessel potentials for $d \geq 2$. It is interesting to note that when $\mathcal{K}(x)=\mathcal{N}(x)$ is Newtonian, $\mathcal{N}(x)=C_{d}|x|^{-(d-2)}$ for $d \geq 3$ or $\mathcal{N}(x)=-C \log (|x|)$ for $d=2$, we recover in (1.1)-(1.3) the classical parabolic-elliptic Patlak-Keller-Segel (PKS) model of chemotaxis:

$$
\left\{\begin{array}{l}
\partial_{t} u+\nabla \cdot(u \nabla c)=\Delta A(u), \text { in } \Omega \times(0, \infty), \\
c=\mathcal{N} * u, \text { in } \Omega \times(0, \infty)
\end{array}\right.
$$


that is, the second equation in (1.4) reads $-\Delta c=u$. We could not find a proof for the existence of the global attractor, and its properties for the PKS model (1.4) in the literature. Our results cover this important case as well. For the precise statements of the results, we refer the reader to sections 3-4. Another basic system of equations modelling chemotaxis was established in the 1970's by Keller and Segel [34, 35, 36]. In these systems, the population concentration $u$ satisfies the first equation of (1.4), while the chemotactic agent $c$ satisfies instead the parabolic equation

$$
\tau \partial_{t} c-\Delta c=\beta_{1} c+\beta_{2} u, \text { in } \Omega \times(0, \infty),
$$

for some real constants $\beta_{1}, \beta_{2}$, and $\tau>0$. The latter system is usually referred in the literature as the parabolic-parabolic Patlak-Keller-Segel model of chemotaxis. The mathematical and biological literature, concerned primarily with the qualitative properties of the solutions to the parabolic-parabolic PKS (and some of its generalizations), is quite extensive and much of the work before 2003 is largely referenced in the survey papers by Horstmann [40, 41]. More recent results pertaining to the long-term behavior of solutions, in terms of global and exponential attractors, of the parabolic-parabolic PKS model can be found in $[1,2,3,25]$. The issue of the convergence to single stationary states as time goes to infinity is also addressed in [26, 54]. Finally, it is worth observing that the parabolic-elliptic PKS model (1.4) follows for an appropriate choice of the parameters $\beta_{1}$ and $\beta_{2}$, not only as a formal limit but also rigorously as $\tau \rightarrow 0^{+}$, from the parabolic-parabolic PKS model [6].

Now, we wish to provide the reader with some further background on the above system (1.1)-(1.3). The whole issue of well-posedness of weak solutions for equation (1.1) with no-flux boundary conditions on a bounded convex domain of class $\mathcal{C}^{1}$, and sufficiently smooth interaction kernels $\mathcal{K}$ (see below for the precise assumptions), was established in [12, 17] (see also [7] for related results). When $\mathcal{K}$ is not smooth enough, finite time blowup of some solutions can occur (see, for instance, [13, 14, $15,16,17])$. However, it is worth emphasizing that in population dynamics, the non-local effects are generally modelled with smooth, fast-decaying kernels. When problem (1.1)-(1.3) is uniformly parabolic (i.e., $A^{\prime}(y) \geq \varepsilon>0$, for all $y \geq 0$ ), we can improve our analysis from degenerate diffusion. More precisely, we give a complete characterization of the global attractor $\mathcal{A}_{\varepsilon}$ in this case, as the union of all unstable manifolds generated by all equilibria (steady-state) solutions of the non-degenerate aggregation equation (see Section 4, Theorem 4.6). At this point one could argue that the long-time behavior of the system (1.1)-(1.3) with nondegenerate diffusion $\left(A^{\prime}(y) \geq \varepsilon>0\right)$ is properly described by the global attractor. However, it is wellknown that the global attractor can present several drawbacks, among which we can mention that it may only attract the trajectories at a slow rate, and that it may miss important transient behaviors because the global attractor consists only of states in a final stage. This phenomenon is already present for models of pattern formation in chemotaxis (see [52]). Another suitable object which contains the global attractor, and thus is more rich in content than the global attractor is the so-called exponential attractor (see [45, 52]; cf. also Section 4). In Section 4 we show that the problem (1.1)-(1.3) with nondegenerate diffusion $\left(A^{\prime}(y) \geq \varepsilon>0\right)$ admits also an exponential attractor $\mathcal{M}_{\varepsilon}$ (and as a result, the global attractor $\mathcal{A}_{\varepsilon}$ is finite-dimensional), globally bounded in $C^{\alpha}(\bar{\Omega})$, for some $\alpha \in(0,1)$, provided that we additionally assume that the kernel $\mathcal{K}$ is sufficiently smooth at the origin, i.e.,

$$
\mathcal{K} \in W^{2,1}\left(B_{1}(0)\right)
$$


Here $B_{1}(0) \subset \mathbb{R}^{d}$ is the ball centered at the origin and radius equal to one.

In the final section, we also discuss the convergence of any bounded solutions $u(t)$ of the non-degenerate aggregation equation to single steady states, provided that $\Phi$ is a real analytic function on $\mathbb{R}_{+}$, where $\Phi^{\prime \prime}(y):=A^{\prime}(y) / y$ and $\Phi(0)=\Phi^{\prime}(1)=0$. Moreover, we also establish the convergence rate of the bounded solution $u(t)$ to a single steady state $u_{*}$ :

$$
\left\|u(t)-u_{*}\right\|_{L^{p}(\Omega)} \sim(1+t)^{-\rho}, \text { as } t \rightarrow \infty,
$$

for any $p>1$, and some $\rho=\rho\left(u_{*}, p\right) \in(0,1)$ (see Theorem 5.1). We refer the reader to Section 5 for the precise assumptions, statements, and further discussions.

As noted in [53], the system (1.1)-(1.3) exhibits interesting coarsening dynamics whose behavior is similar to another well-known (non-biological) model, the so-called nonlocal Cahn-Hilliard equation, which also exhibits behaviors in which small localized clumps form and merge into larger clumps over time. The latter equation has also been recently studied in $[10,11,29,30,38,43]$. We refer the reader for more related results concerning the nonlocal Cahn-Hilliard equation to [28], where a complete characterization of the long-term behavior is also given for this equation. Our proofs for the existence of attractors and their properties will explore various connections which exist between the aggregation equation (1.1) and the non-local Cahn-Hilliard equation (see [28], and references therein).

\section{Weak solutions}

We begin with some basic notations and preliminaries. Throughout the section, $C \geq 0$ will denote a generic constant, while $Q: \mathbb{R}_{+} \rightarrow \mathbb{R}_{+}$will denote a generic increasing function. All these quantities, unless explicitly stated, are independent of time, an approximation parameter $\varepsilon>0$, and the initial data. Further dependencies of these quantities will be specified on occurrence. In the sequel, our investigation will be mainly divided into two cases:

- (i) $\sigma\left(\Gamma_{D}\right)=0$, i.e., $\Gamma_{D}$ is empty, and

- (ii) $\sigma\left(\Gamma_{D}\right)>0$, i.e., $\Gamma_{D}$ is an open set of positive $\sigma$-measure,

where we recall that $\sigma$ denotes the restriction to $\partial \Omega$ of the $(d-1)$-dimensional Hausdorff measure.

Following [12, Section 2], [17, Definition 1] we make the following assumptions on $A$ and $\mathcal{K}$ :

H1 The domain $\Omega \subset \mathbb{R}^{d}, d \geq 1$, is convex and of class $\mathcal{C}^{1}$.

H2 $A \in C^{1}[0, \infty), A(0)=0$, and there exists constants $C_{A}, \bar{C}_{A}>0$ such that

$$
C_{A} y^{m-1} \leq A^{\prime}(y) \leq \bar{C}_{A} y^{m-1}, \forall y \geq 0
$$

for some $m>m_{*}$, with

$$
m_{*}:= \begin{cases}1+\frac{1}{\gamma}-\frac{2}{d} \geq 1, & \text { if } \sigma\left(\Gamma_{D}\right)=0, \\ 2, & \text { if } \sigma\left(\Gamma_{D}\right)>0,\end{cases}
$$

for any $1 \leq \gamma \leq \frac{d}{2}$. For $\sigma\left(\Gamma_{D}\right)=0, m_{*}$ is the critical exponent defined in [17, Definition 5 and Lemma 10].

H3 $\mathcal{K} \in W_{\text {loc }}^{1,1}\left(\mathbb{R}^{d}\right) \cap C^{3}\left(\mathbb{R}^{d} \backslash\{0\}\right)$ satisfies the following assumptions:

(i) $\mathcal{K}$ is symmetric, $\mathcal{K}(x)=k(|x|)$ and $k$ is nonincreasing. 
(ii) $k^{\prime \prime}(r)$ and $k^{\prime}(r) / r$ are monotone on $r \in(0, \delta)$, for some $\delta>0$.

(iii) $\left|D^{3} \mathcal{K}(x)\right| \leq C|x|^{-d-1}$, for some $C>0$.

Note that since the function $k$ in condition (H3) is nonincreasing, the nonlocal term in (1.1) models attraction. Moreover, these conditions imply that if $\mathcal{K}$ is singular, the singularity is restricted to the origin, so that both the Newtonian and Bessel potentials for $d \geq 2$ are included in our analysis. Finally, assumption (H2) implies that our problem (1.1)-(1.3) is subcritical in the terminology of [17, Definition 6], and note that the total population $u$ is preserved in time. In particular, there holds

$$
\langle u(t)\rangle:=\frac{1}{|\Omega|} \int_{\Omega} u(t, x) d s=\left\langle u_{0}\right\rangle, \text { for all } t \geq 0 .
$$

Here, $|\Omega|$ denotes the $d$-dimensional Lebesgue measure of $\Omega$. This property is lost whenever $\Gamma_{D}$ is nonempty, $\sigma\left(\Gamma_{D}\right)>0$, and $A$ is non-degenerate such that $A^{\prime}(s) \geq \varepsilon>0$.

Let us recall some important properties of the kernel $\mathcal{K}$, proven in $[17$, Section 1.3], which imply a useful number of estimates in weak $L^{p, \infty}$-spaces, with quasi-norm

$$
\|u\|_{L^{p, \infty}}=\sup _{\alpha>0}\left(\alpha^{p} \lambda_{\alpha}(u)\right)^{1 / p}
$$

where $\lambda_{\alpha}(u):=|\{u>\alpha\}|$ is the distribution function of $u$ (see [17, Lemmas 1-3]).

Lemma 2.1. Let $\mathcal{K}$ satisfy Assumption (H3), (i)-(iii).

(a) There holds $\nabla \mathcal{K} \in L^{\frac{d}{d-1}, \infty}(\Omega)$. If $d \geq 3$, then $\mathcal{K} \in L^{\frac{d}{d-2}}, \infty(\Omega)$.

(b) Let $\vec{V}=\nabla \mathcal{K} * u$. Then, for any $1<p<\infty$, there exists a constant $C=C(p)>0$ such that

$$
\|\nabla \vec{V}\|_{L^{p}(\Omega)} \leq C\|u\|_{L^{p}(\Omega)} .
$$

(c) Let $d \geq 3$. Suppose that $\gamma \in(1, d / 2)$. Then $\mathcal{K} \in L_{\text {loc }}^{\frac{d}{d \gamma-2}, \infty}(\Omega)$ if and only if $D^{2} \mathcal{K} \in L_{\text {loc }}^{\gamma, \infty}(\Omega)$. The same holds for $\nabla \mathcal{K} \in L_{\text {loc }}^{\frac{d}{d \gamma-1}, \infty}(\Omega)$.

REMARK 2.1. The above lemma shows that every admissible kernel $\mathcal{K}$ that satisfies (H3), (i)-(iii), is at least as integrable as the Newtonian potential.

As in [12, Definition 2.1] and [17, Definition 3], our notion of bounded, nonnegative weak solutions to (1.1)-(1.3) is as follows. By $H_{D}^{1}(\Omega)$ we denote the space of all functions $u \in H^{1}(\Omega)$ such that $u=0$ on $\Gamma_{D}$, if $\sigma\left(\Gamma_{D}\right)>0$, and by $\left(H_{D}^{1}(\Omega)\right)^{*}$ the dual of $H_{D}^{1}(\Omega)$. Below, the space $H_{D}^{1}(\Omega)$ is replaced by $H^{1}(\Omega)$ whenever $\sigma\left(\Gamma_{D}\right)=0$ (i.e., if $\Gamma_{D}$ is empty).

Definition 2.2. Let $T>0$ be given, but otherwise arbitrary. A function $u: \Omega \times$ $[0, T] \rightarrow[0, \infty)$ is a weak solution of (1.1)-(1.3) if

$$
\begin{aligned}
u & \in L^{\infty}(\Omega \times[0, T]), A(u) \in L^{2}\left(0, T ; H_{D}^{1}(\Omega)\right), \\
\partial_{t} u & \in L^{2}\left(0, T ;\left(H_{D}^{1}(\Omega)\right)^{*}\right), u \nabla \mathcal{K} * u \in L^{2}(\Omega \times[0, T]),
\end{aligned}
$$

and the following identity holds:

$$
\left\langle\partial_{t} u(t), w\right\rangle_{\left(H_{D}^{1}(\Omega)\right)^{*}, H_{D}^{1}(\Omega)}+\int_{\Omega} \nabla A(u(t)) \cdot \nabla w-u(t)(\nabla \mathcal{K} * u(t)) \cdot \nabla w d x=0,
$$


for all $w \in H_{D}^{1}(\Omega)$, for almost all $t \in[0, T]$.

REMARK 2.2. Note that by $(2.2), u \in C\left(0, T ;\left(H_{D}^{1}(\Omega)\right)^{*}\right)$ such that the initial condition in (1.3) is understood in the weak sense of $\left(H_{D}^{1}(\Omega)\right)^{*}$. In fact, in [12, Section 2] it is shown that $u \in C\left(0, T ; L^{p}(\Omega)\right)$, for all $1<p<\infty$, provided that $0 \leq u_{0} \in L^{\infty}(\Omega)$, so that the initial condition is also satisfied in the $L^{p}$-sense.

We have the following result concerning well-posedness of the system (1.1)-(1.3).

THEOREM 2.3. Let the assumptions (H1)-(H3) be satisfied in both cases (i) $+($ ii) (i.e., $\sigma\left(\Gamma_{D}\right) \geq 0$ ), and assume $0 \leq u_{0} \in L^{\infty}(\Omega)$. Then there exists a unique (global) nonnegative solution to problem (1.1)-(1.3) in the sense of Definition 2.2. Moreover, every weak solution satisfies the following dissipative inequality:

$$
\mathcal{E}(u(t))+\int_{0}^{t} \int_{\Omega} u(s)\left|\nabla \Phi^{\prime}(u(s))-\nabla \mathcal{K} * u(s)\right|^{2} d x d s \leq \mathcal{E}\left(u_{0}\right),
$$

for all $t \geq 0$, where

$$
\mathcal{E}(u(t)):=\int_{\Omega} \Phi(u(t)) d x-\frac{1}{2} \int_{\Omega} \int_{\Omega} u(x, t) \mathcal{K}(x-y) u(y, t) d x d y,
$$

and $\Phi$ is strictly convex on $(0, \infty)$ such that $\Phi^{\prime \prime}(y):=A^{\prime}(y) / y$ and $\Phi(0)=\Phi^{\prime}(1)=0$.

Proof. We only briefly mention the main steps in the proof. The uniqueness of weak solutions follows from [12, Theorem 2.4] (cf. also [17, Theorem 3] for a more general result) with some minor modifications (see Lemma 2.4 below) since the boundary terms involving convolutions with the kernel $\mathcal{K}$ vanish on $\Gamma_{D}$, if $\sigma\left(\Gamma_{D}\right)>0$.

Step 1 (Local existence). The local existence result can be carried out in a standard manner by first regularizing $A(y)$ with $A_{\varepsilon}(y)=A(y)+\varepsilon y, \forall \varepsilon>0, \mathcal{K}$ by a sequence of smooth kernels $\mathcal{J}_{\varepsilon} \mathcal{K}$ (where $\mathcal{J}_{\varepsilon}$ is a standard mollifier), and then the initial data $u_{\varepsilon}(0)=u_{0, \varepsilon} \in C^{\infty}(\Omega) \cap C^{1}(\bar{\Omega})$, followed by passage to limit as $\varepsilon \rightarrow 0^{+}$in the corresponding regularized problem $P_{\varepsilon}$ :

$$
\begin{cases}\partial_{t} u_{\varepsilon}+\nabla \cdot\left(u_{\varepsilon} \nabla \mathcal{J}_{\varepsilon} \mathcal{K} * u_{\varepsilon}\right)=\Delta A_{\varepsilon}\left(u_{\varepsilon}\right), & \text { in } \Omega \times(0, \infty), \\ \left(\nabla A_{\varepsilon}\left(u_{\varepsilon}\right)-u_{\varepsilon} \nabla \mathcal{J}_{\varepsilon} \mathcal{K} * u_{\varepsilon}\right) \cdot \vec{n}=0, & \text { on } \Gamma_{N} \times(0, \infty), \\ u_{\varepsilon}=0, & \text { on } \Gamma_{D} \times(0, \infty),\end{cases}
$$

(see [12, Theorem 2.13], [17, Section 3]). This is achieved by proving a series of uniform (in $\varepsilon>0$ ) estimates for the approximate solutions $u_{\varepsilon}(t)$, in particular, by establishing the uniform bound in $L^{\infty}(\Omega)$-norm:

$$
\sup _{t \geq 0}\left\|u_{\varepsilon}(t)\right\|_{L^{\infty}(\Omega)} \leq C,
$$

for some positive constant $C$ which is independent of $\varepsilon>0$ (and even the times $t, T$ ). This norm gives further uniform estimates (with respect to $\varepsilon>0$ ) for solutions in the normed spaces of (2.2). More precisely, we can obtain the following bounds:

$$
\begin{aligned}
\left\|A\left(u_{\varepsilon}\right)\right\|_{L^{2}\left(0, T ; H_{D}^{1}(\Omega)\right)} & \leq C, \\
\left\|\partial_{t} u\right\|_{L^{2}\left(0, T ;\left(H_{D}^{1}(\Omega)\right)^{*}\right)} & \leq C, \\
\|u \nabla \mathcal{K} * u\|_{L^{2}(\Omega \times[0, T])} & \leq C,
\end{aligned}
$$


for some positive constant $C$ independent of $\varepsilon$. Moreover, for every $\varepsilon>0$ it holds that

$$
\left\|u_{\varepsilon}\right\|_{L^{2}\left(0, T ; H_{D}^{1}(\Omega)\right)} \leq \frac{C}{\sqrt{\varepsilon}} .
$$

Thus, on account of the above uniform estimates the sequence of solutions $u_{\varepsilon}$ is precompact in $L^{p}(\Omega \times[0, T])$, for all $1 \leq p<\infty$, (cf. [17, Lemma 9], [12]), and we can pass to the limit as $\varepsilon \rightarrow 0$ in the nonlinear terms in (2.6). In particular, it can be shown that

$$
A\left(u_{\varepsilon}(t)\right) \rightarrow A(u(t)) \text { weakly in } L^{2}\left(0, T ; H_{D}^{1}(\Omega)\right)
$$

and

$$
u_{\varepsilon} \nabla \mathcal{J}_{\varepsilon} \mathcal{K} * u_{\varepsilon} \rightarrow u \nabla \mathcal{K} * u \text { weakly in } L^{2}(\Omega \times[0, T])
$$

(see [17, Theorem 1]). Let us now comment how to get the estimates (2.7)-(2.11) in each of the following cases: $\sigma\left(\Gamma_{D}\right)=0$ or $\sigma\left(\Gamma_{D}\right)>0$.

- Case (i): $\sigma\left(\Gamma_{D}\right)=0$ (i.e., $\Gamma_{D}$ is empty). The argument for deducing (2.7) relies on showing that the $L^{1}$-norm of $u_{\varepsilon}(t)$ (see (2.1)) a priori controls the $L^{p}$-norm for $1<p<\infty$, and then that the latter norm controls the $L^{\infty}$-norm of $u_{\varepsilon}(t)$; see (2.7) (cf. also [17, Lemma 8]). The Lemma 2.1 is crucial for the proof of the uniform bound (2.7). Under the assumptions (H1)-(H3) the bounds (2.7)-(2.11) were obtained in [17, Section 3] (cf. also [12]).

- Case (ii): $\sigma\left(\Gamma_{D}\right)>0$. The arguments from [17, 12] leading to estimates (2.7)(2.10) on intervals $(0, T)$, for some $T=T(p)>0$, seem to break down if $\Gamma_{D}$ is nonempty and $\sigma\left(\Gamma_{D}\right)>0$ since there is no conservation of mass (2.1) for (2.6) in this case; hence, the $L^{1}$-norm of $u_{\varepsilon}(t)$ is not controlled a priori. However, even in this case, the same proof of obtaining local-in-time estimates for $u_{\varepsilon}(t)$ in [17, Lemma 8] applies, but we need to use the inequality

$$
\left\|u_{\varepsilon}(t)\right\|_{L^{p}(\Omega)}^{p} \leq 2^{p}\left(\left\|\left(u_{\varepsilon}(t)-k\right)_{+}\right\|_{L^{p}(\Omega)}^{p}+k^{p}|\Omega|\right) \text {, for any } k>0,
$$

instead of the usual one [17, Inequality (25)]. Then, by arguing as in $[17$, Inequality (27) and Lemma 8, Step 2] (note that boundary terms involving convolutions with the kernel $\mathcal{K}$ vanish on $\Gamma_{D}$, if $\sigma\left(\Gamma_{D}\right)>0$; also now the last constant on the right-hand side of [17, Inequality (27)] depends only on $k$ and $p$, and not on $L^{1}$-norm of $u_{\varepsilon}(0)$ ), we also obtain a (uniform in $\varepsilon>0$ ) $L^{\infty}$-bound for $u_{\varepsilon}(t), t \in[0, T]$, for some $T=T(p)>0$.

By virtue of these observations, the proof of the local existence argument in both cases (i)+(ii) goes exactly as in [17, Section 3] (cf. also [12, Section 2.2]). A proof of the energy inequality (2.4) can be found in [12, Lemma 5.1], [17, Proposition 1]. Continuation of the weak solutions is a straightforward consequence of the local existence theory (in Step 1) and the proof of [17, Theorem 4].

Step 2 (Global existence).

- First, let $\sigma\left(\Gamma_{D}\right)=0$. Since the problem is subcritical by assumption (H2), the global existence result follows from [17, Lemma 10 and Remark 9], which shows that (2.7) is also satisfied uniformly with respect to $t, T$.

- When $\sigma\left(\Gamma_{D}\right)>0$, it suffices to establish a uniform (in $\varepsilon>0$ and time $t, T>0$ ) $L^{p}$-estimate for $u_{\varepsilon}(t)$ and to exploit an argument similar to [17, Lemma 8 , 
Step 2] to deduce the uniform (with respect to $t, T, \varepsilon$ ) bound (2.7). However, the key difference with respect to the case $\sigma\left(\Gamma_{D}\right)=0$ is that mass is not conserved for (2.6); hence, the $L^{1}$-norm of $u_{\varepsilon}(t)$ is not controlled a priori and we need a different argument to deduce the uniform bound in $L^{p}$-norm for $u_{\varepsilon}(t)$; see Proposition 3.1 and Remark 3.1 below. The latter bound requires that the critical exponent $m_{*}=2$ in (H2).

The proof of Theorem 2.3 is now complete.

REMARK 2.3. In order to establish the energy identity in (2.4), it would suffice to show that $\Phi^{\prime}(u) \in L^{2}\left(0, T ; H_{D}^{1}(\Omega)\right)$ so we can test equation $(2.3)$ with $\Phi^{\prime}(u)$ and $\mathcal{K} * u$, respectively. At the moment, this seems unreachable for our problem with a degenerate diffusion function $A(u) \sim u^{m}$, like in (H2). However, note that $\Phi(u) \in L^{2}\left(0, T ; H_{D}^{1}(\Omega)\right), \Phi \in L^{\infty}\left(0, T ; L^{1}(\Omega)\right)$ since $\Phi(u) \sim A(u)$, by (H2). When the diffusion $A$ in (1.1) is non-degenerate (say, like in problem (2.6)), we can establish the energy identity (see the proof of Theorem 2.3). Indeed, in this case we can easily check that $\Phi^{\prime}\left(u_{\varepsilon}\right) \in L^{2}\left(0, T ; H_{D}^{1}(\Omega)\right), \forall \varepsilon>0$, on account of (2.7) and (2.11). Thus, the key multiplication of the corresponding weak formulation associated with (2.6) (see (2.3)) with $\Phi^{\prime}\left(u_{\varepsilon}\right)$ and $\mathcal{K} * u_{\varepsilon} \in L^{2}\left(0, T ; H_{D}^{1}(\Omega)\right)$, respectively, is allowed. Exploiting, for instance, [12, Lemma 2.6], and owing to the convexity of $\Phi_{\varepsilon}$, we get equality in (2.4) for the energy $\mathcal{E}_{\varepsilon}\left(u_{\varepsilon}(t)\right)$, associated with (2.6), which is defined by

$$
\mathcal{E}_{\varepsilon}\left(u_{\varepsilon}(t)\right):=\int_{\Omega} \Phi_{\varepsilon}\left(u_{\varepsilon}(t)\right) d x-\frac{1}{2} \int_{\Omega} \int_{\Omega} u_{\varepsilon}(x, t) \mathcal{K}(x-y) u_{\varepsilon}(y, t) d x d y
$$

( $\Phi_{\varepsilon}$ is the same function as above, but with $A$ replaced by $A_{\varepsilon}$ ).

The next lemma gives the (Hölder) continuity of solutions with respect to the initial data in $\left(H_{D}^{1}(\Omega)\right)^{*}$ and $\left(H^{1}(\Omega)\right)^{*}$, respectively.

Lemma 2.4. Let the hypotheses of Theorem 2.3 be satisfied. Let $u_{1}(t)$ and $u_{2}(t)$ be any two weak solutions of (1.1)-(1.3) corresponding to any two initial data $u_{01}$ and $u_{02}$, respectively. If $\sigma\left(\Gamma_{D}\right)=0$, we further take $\left\langle u_{01}\right\rangle=M_{1}$ and $\left\langle u_{02}\right\rangle=M_{2}$, for some $M_{1}, M_{2} \geq 0$. For all $t \in[0, T]$, the following estimates hold:

$$
\left\|u_{1}(t)-u_{2}(t)\right\|_{\left(H_{D}^{1}(\Omega)\right)^{*}} \leq C\left(\left\|u_{10}-u_{20}\right\|_{\left(H_{D}^{1}(\Omega)\right)^{*}}\right)^{e^{-C t}}, \text { if } \sigma\left(\Gamma_{D}\right)>0,
$$

and

$$
\left\|u_{1}(t)-u_{2}(t)\right\|_{\left(H^{1}(\Omega)\right)^{*}} \leq C\left(\left\|u_{10}-u_{20}\right\|_{\left(H^{1}(\Omega)\right)^{*}}+\left|\left\langle u_{01}-u_{20}\right\rangle\right|\right)^{e^{-C t}},
$$

if $\sigma\left(\Gamma_{D}\right)=0$.

Proof. We briefly explain how to get (2.15); the estimate (2.14) is similar. Let $u(t):=u_{1}(t)-u_{2}(t)$ and observe that (2.1) yields $\langle u(t)\rangle=M_{1}-M_{2}=: M_{12}$, for all $t \geq 0$, but $M_{12} \neq 0$, in general. As in [12, Theorem 2.4], [17, Section 2], consider the Neumann problem

$$
\begin{cases}\Delta \phi(t)=u(t)-M_{12}, & \text { in } \Omega \\ \nabla \phi(t) \cdot \vec{n}=0, & \text { on } \Gamma,\end{cases}
$$

for which $\langle\phi(t)\rangle=0$. Notice that since $\langle u(t)\rangle=M_{12},(2.16)$ has a solution. Consider the operator $A_{N}=-\Delta_{N}$, with domain $D\left(A_{N}\right)=\left\{\varphi \in H^{2}(\Omega):(\nabla \varphi \cdot \vec{n})_{\mid \Gamma}=0\right\}$. Clearly $\phi(0)=A_{N}^{-1}\left(u(0)-M_{12}\right)$ and recall that, due to a Poincaré inequality,

$$
\|u\|_{\left(H^{1}(\Omega)\right)^{*}}^{2}=\left\|A_{N}^{-1 / 2}(u-\langle u\rangle)\right\|_{L^{2}(\Omega)}^{2}+\langle u\rangle^{2} .
$$


Next, we see that $\partial_{t} \phi(t)$ also satisfies (in the generalized sense) the problem

$$
\begin{cases}\Delta \partial_{t} \phi(t)=\partial_{t} u(t), & \text { in } \Omega, \\ \nabla \partial_{t} \phi(t) \cdot \vec{n}=0, & \text { on } \Gamma\end{cases}
$$

and

$$
\phi \in L^{\infty}(\Omega \times[0, T]) \cap C\left(0, T ; H_{D}^{1}(\Omega)\right), \nabla \phi \in L^{\infty}(\Omega \times[0, T]) .
$$

Thus, arguing in a standard way as in [12, (6)-(12)], we obtain for

$$
\eta(t):=\|u(t)\|_{\left(H^{1}(\Omega)\right)^{*}}^{2}=\|\nabla \phi(t)\|_{L^{2}(\Omega)}^{2}+\left(M_{12}\right)^{2}
$$

the following equality:

$$
\begin{aligned}
\frac{1}{2} \frac{d}{d t} \eta(t) & =\left\langle\nabla \phi(t), \partial_{t} \nabla \phi(t)\right\rangle \\
& =-\left\langle\partial_{t} u(t), \phi(t)\right\rangle=I_{1}+I_{2}+I_{3},
\end{aligned}
$$

where

$$
\begin{aligned}
& I_{1}:=\int_{\Omega} \nabla A\left(u_{1}(t)\right)-\nabla A\left(u_{2}(t)\right) \cdot \nabla \phi(t) d x, \\
& I_{2}:=-\int_{\Omega}(u(t)) \nabla \mathcal{K} * u_{1}(t) \cdot \nabla \phi(t) d x, \\
& I_{3}:=-\int_{\Omega} u_{2}(t) \nabla \mathcal{K} *(u(t)) \cdot \nabla \phi(t) d x .
\end{aligned}
$$

Since $A$ is increasing and bounded (i.e., $\left\|A^{\prime}\left(u_{i}\right)\right\|_{L^{\infty}(\Omega)} \leq C$, since $u_{i}$ is bounded), from (2.16) we have

$$
\begin{aligned}
I_{1} & =-\int_{\Omega}\left(A\left(u_{1}\right)-A\left(u_{2}\right)\right)\left(u_{1}-u_{2}\right) d x+M_{12} \int_{\Omega} A\left(u_{1}\right)-A\left(u_{2}\right) d x \\
& \leq C\left(M_{1}-M_{2}\right)^{2} .
\end{aligned}
$$

To bound the $I_{2}, I_{3}$ integral terms, we integrate by parts and proceed as in $[12,17]$. We deduce

$$
\begin{gathered}
I_{2}=-\sum_{i, j} \int_{\Omega} \partial_{i j} \phi \partial_{j} \mathcal{K} * u_{1} \partial_{i} \phi d x-\sum_{i, j} \int_{\Omega} \partial_{i} \phi \partial_{j j} \mathcal{K} * u_{1} \partial_{i} \phi d x \\
+\sum_{i, j} \int_{\Gamma}\left[\left(\partial_{j} \mathcal{K} * u_{1}\right) n_{j}\right]\left|\partial_{i} \phi\right|^{2} d \sigma .
\end{gathered}
$$

The last term on the right-hand side of (2.21) is nonpositive since $\Omega$ is convex and $\mathcal{K}$ is radially decreasing (i.e., as in [12], we have $\left(\nabla \mathcal{K} * u_{1}\right) \cdot \vec{n} \leq 0$ on $\left.\Gamma_{N}\right)$. Integration by parts in the first term gives the bound

$$
\sum_{i, j} \int_{\Omega} \partial_{i j} \phi \partial_{j} \mathcal{K} * u_{1} \partial_{i} \phi d x \leq-\frac{1}{2} \int_{\Omega}\left(\Delta \mathcal{K} * u_{1}\right)|\nabla \phi|^{2} d x
$$

which together with (2.21) entails

$$
I_{2} \leq C \int_{\Omega}\left|D^{2} \mathcal{K} * u_{1}\right||\nabla \phi|^{2} d x
$$


for some positive constant $C$ which only depends implicitly on the uniformly controlled $L^{p}$-norms of $u_{1}$ and $u_{2}$. Arguing as in [17, (17)], by using Lemma 2.1-(b) and since $\left(M_{12}\right)^{2} \leq \eta(t)$, we deduce for any $p \geq 2$ that

$$
I_{2} \leq C p \eta(t)^{1-1 / p} \text { and } I_{3} \leq C\left\|u_{2}\right\|_{L^{\infty}(\Omega)} \eta(t) .
$$

Consequently, we obtain the differential inequality

$$
\frac{d}{d t} \eta(t) \leq C p \eta(t)^{1-1 / p}+C \eta(t), \forall t \in[0, T] .
$$

As in [18, Theorem 3.3], the idea is to fix $p=p(\eta(t))$ in an optimal way. To this end, choose a sufficiently large constant $\widetilde{C}>0$ such that $p=\log (\widetilde{C} / \eta(t)) \geq 2$, for all $t \in[0, T]$. First, recall that $\eta(t)$ is bounded on $t \in[0, T]$ and that the first term on the right-hand side of (2.24) dominates the second one since we need the estimate for $\eta(t)$ small only. Thus, the second term on the right-hand side is not essential and we can derive the following differential inequality:

$$
\frac{d}{d t} \eta(t) \leq C \eta(t) \log \left(\frac{\widetilde{C}}{\eta(t)}\right), \forall t \in[0, T]
$$

Here, we have used the elementary inequality $\eta(t)^{-1 / p} \leq C$, for the $p$ chosen above. Integrating (2.25) with respect to $t \in(\delta, s)$, we obtain

$$
\eta(s) \leq \widetilde{C}\left[\frac{\eta(\delta)}{\widetilde{C}}\right]^{e^{-C(s-\delta)}}, \forall s \in(0, T] .
$$

Passing to the limit as $\delta \rightarrow 0$ in (2.26), recalling that $\eta(t)$ is continuous, we get the desired estimate (2.15).

\section{Optimal regularity and the global attractor}

In this section, we derive several uniform estimates for the solutions of the problem (1.1)-(1.2) which are necessary for the study of the asymptotic behavior as time goes to infinity. In a first step, we obtain dissipative estimates for solutions in the spaces $L^{p}, L^{\infty}$, and $C^{\alpha}, \alpha>0$, uniformly with respect to time and the initial data. Incidently, the estimates derived below allow one also to obtain optimal regularity results for the weak solutions on $\Omega \times[\tau, \infty)$, for every $\tau>0$, associated with the system (1.1)-(1.3). Finally, the a priori estimates will be deduced by a formal argument, which can be justified rigorously by means of the approximation procedure devised in $[12$, Section $2]$, [17] by means of (2.6). Regardless of the type of approximation procedure being used, the regularity properties

$$
u_{\varepsilon} \in L^{\infty}\left(0, T ; L^{\infty}(\Omega)\right) \cap L^{2}\left(0, T ; H_{D}^{1}(\Omega)\right), \quad \forall \varepsilon, T>0
$$

are essential in order to rigorously perform these computations. To this end, we shall only perform our (formal) computations to the original system (1.1)-(1.3), for the sake of simplicity.

The (uniform) dissipative $L^{p}$-estimate when $\sigma\left(\Gamma_{D}\right)>0$ is different than the estimate when $\sigma\left(\Gamma_{D}\right)=0$ (in this case, it was obtained in [17, Lemma 10 and Remark $9]$ ), since in the former case there is no conservation of mass in (2.6). It is given by the following. 
Proposition 3.1. Let the assumptions (H1)-(H3) be satisfied and assume that $\sigma\left(\Gamma_{D}\right)>0$ (Case (ii)). Then there exists constants $C_{*}, C_{+}>0$, and $\mu>1$, independent of time and the initial data, such that every weak solution of (1.1)-(1.3) satisfies

$$
\|u(t)\|_{L^{p}(\Omega)}^{p} \leq\left(\frac{C_{*}}{C_{+}}\right)^{\frac{1}{\mu}}+\left[C_{+}(\mu-1) t\right]^{-\frac{1}{\mu-1}}, \text { for all } t \geq \tau>0,
$$

provided that $p>m+2 d /(d-1)$. The constants $C_{*}, C_{+}$, and $\mu$ can be computed explicitly in terms of the physical parameters of the problem.

Proof. We first begin by noting that $\nabla \mathcal{K} \in L^{q, \infty}(\Omega)$, for any $q>d /(d-1)$, by Lemma 2.1,

(a). This yields

$$
\nabla \mathcal{K} \mathbf{1}_{B_{1}(0)} \in L^{1}\left(\mathbb{R}^{d}\right) \text { and } \nabla \mathcal{K} \mathbf{1}_{\mathbb{R}^{d} \backslash B_{1}(0)} \in L^{q}\left(\mathbb{R}^{d}\right),
$$

for any $q>d /(d-1)$. Next, let $\varphi \in C^{1}(\bar{\Omega}) \cap C^{2}(\Omega)$ and $\lambda$ denote the principal eigenfunction and eigenvalue of

$$
-\Delta \varphi=\lambda \varphi \text { in } \Omega
$$

with the boundary condition

$$
\nabla \varphi \cdot \vec{n}=0 \text { on } \Gamma_{N}
$$

such that

$$
\nabla \varphi \cdot \vec{n}+\varphi=0 \text { on } \Gamma_{D}
$$

Clearly, $\lambda>0$ and by the maximum principle (see, e.g., [23]) $\varphi>0$ in $\bar{\Omega}$ since $\sigma\left(\Gamma_{D}\right)>0$. Without loss of generality, we may assume that $\|\varphi\|_{L^{1}(\Omega)}=1$.

Set now $a(y):=A^{\prime}(y)$. Testing equation (2.3) by $p u^{p-1} \varphi, p>1$, we obtain

$$
\begin{aligned}
\frac{d}{d t} \int_{\Omega} u^{p}(t) \varphi d x= & -p \int_{\Omega} a(u(t)) \nabla u(t) \cdot \nabla\left(u^{p-1}(t) \varphi\right) d x \\
& \quad+p \int_{\Omega} u(t)(\nabla \mathcal{K} * u(t)) \nabla\left(u^{p-1}(t) \varphi\right) d x \\
= & I_{1}+I_{2}+I_{3}+I_{4},
\end{aligned}
$$

where

$$
\begin{aligned}
& I_{1}:=-p(p-1) \int_{\Omega} a(u) u^{p-2}|\nabla u|^{2} \varphi(x) d x, \\
& I_{2}:=-p \int_{\Omega} a(u) u^{p-1} \nabla u \cdot \nabla \varphi(x) d x, \\
& I_{3}:=p(p-1) \int_{\Omega} u(\nabla \mathcal{K} * u) \cdot \nabla u\left(u^{p-2} \varphi(x)\right) d x, \\
& I_{4}:=p \int_{\Omega} u(\nabla \mathcal{K} * u) \cdot \nabla \varphi(x) u^{p-1} d x .
\end{aligned}
$$

First, from (H2) it is easy to see that

$$
I_{1} \leq-C p(p-1) \int_{\Omega} u^{p-3+m}|\nabla u|^{2} \varphi(x) d x \leq 0 .
$$


On the other hand, setting $\widetilde{a}(u)=\int_{0}^{u} a(y) y^{p-1} d y \geq \frac{C_{A}}{m+p-1} u^{m+p-1}$, and using the definition of $\varphi$ from (3.4)-(3.6), we have

$$
\begin{aligned}
I_{2}= & -p \int_{\Omega} \nabla \widetilde{a}(u) \cdot \nabla \varphi(x) d x=p \int_{\Omega} \widetilde{a}(u) \Delta \varphi(x) d x \\
& \quad-p \int_{\Gamma_{D}} \widetilde{a}(u) \nabla \varphi \cdot \vec{n} d \sigma-p \int_{\Gamma_{N}} \widetilde{a}(u) \nabla \varphi \cdot \vec{n} d \sigma \\
= & -p \lambda \int_{\Omega} \widetilde{a}(u) \varphi(x) d x \\
\leq & -C \lambda \int_{\Omega} u^{m+p-1} \varphi d x,
\end{aligned}
$$

since $\widetilde{a}(0)=0\left(u=0\right.$, a.e. on $\left.\Gamma_{D} \times(0, \infty)\right)$ and $\varphi$ satisfies (3.5). Moreover, we can estimate the integrals $I_{1}, I_{2}$ using the Hölder and Young inequalities as follows:

$$
\begin{aligned}
I_{3} & =p(p-1) \int_{\Omega}\left(u^{\frac{p-m+1}{2}} \sqrt{\varphi}(\nabla \mathcal{K} * u)\right) \cdot\left(\sqrt{\varphi} u^{\frac{p-3+m}{2}} \nabla u\right) d x \\
& \leq p(p-1)\left(\int_{\Omega} u^{p-3+m}|\nabla u|^{2} \varphi(x) d x\right)^{1 / 2}\left(\int_{\Omega}|\nabla \mathcal{K} * u|^{2} u^{p-m+1} \varphi(x) d x\right)^{1 / 2} \\
& \leq \eta p(p-1) I_{1}+C_{\eta} \int_{\Omega}|\nabla \mathcal{K} * u|^{2} u^{p-m+1} \varphi(x) d x
\end{aligned}
$$

for every $\eta>0$. We must once again absorb the last term on the right-hand side of (3.10) into $I_{1}$. For any $q>d /(d-1)$ and $s>1$ such that $1 / s+1 / q=1$, Hölder's inequality yields

$$
\begin{aligned}
C_{\eta} \int_{\Omega}|\nabla \mathcal{K} * u|^{2} u^{p-m+1} d x & \leq C_{\eta}\|\nabla \mathcal{K} * u\|_{L^{2 q}(\Omega)}^{2}\left(\int_{\Omega} u^{(p-m+1) s} d x\right)^{1 / s} \\
& \leq C_{\eta}(\mathcal{K})\|u\|_{L^{2 q}(\Omega)}^{2}\|u\|_{L^{(p-m+1) s}(\Omega)}^{p-m+1},
\end{aligned}
$$

since, from (3.3), there holds

$$
\begin{aligned}
\|\nabla \mathcal{K} * u\|_{L^{2 q}(\Omega)} & \leq\left\|\nabla \mathcal{K} \mathbf{1}_{B_{1}(0)}\right\|_{L^{1}}\|u\|_{L^{2 q}(\Omega)}+\left\|\nabla \mathcal{K} \mathbf{1}_{\mathbb{R}^{d} \backslash B_{1}(0)}\right\|_{L^{2 q}}\|u\|_{L^{1}(\Omega)} \\
& \leq C(\mathcal{K}, \Omega)\|u\|_{L^{2 q}(\Omega)} .
\end{aligned}
$$

Thus, choosing $q, s>1$ in (3.11) in an optimal way such that $2 q=(p-m+1) s>$ $2 d /(d-1)$, we further obtain in (3.10), by virtue of (3.11) and Young's inequality, that

$$
\begin{aligned}
I_{3} & \leq \eta p(p-1) I_{1}+C_{\eta}(\mathcal{K}, \Omega)\|u\|_{L^{p-m+3}(\Omega)}^{p-m+3} \\
& \leq \eta p(p-1) I_{1}+\bar{\eta} \int_{\Omega} u^{m-1+p} \varphi d x+C_{\eta, \bar{\eta}}(\mathcal{K}, \Omega, p, m, \varphi),
\end{aligned}
$$

for every $\bar{\eta}>0$, since $p+m-1>p-m+3$ (recall that $m>2$ ). It follows by choosing sufficiently small $\eta \leq C / 2$ in (3.10), and $\bar{\eta} \leq(C / 2) \lambda$ in (3.13), that the integral term $I_{3}$ can be completely absorbed into $I_{1}$. The term $I_{4}$ can be bounded in exactly the same way. We have

$$
I_{4}=p \int_{\Omega} u(\nabla \mathcal{K} * u) \cdot \nabla \varphi(x) u^{p-1} d x \leq \epsilon \int_{\Omega} u^{p+m-1} \varphi(x) d x+C_{\epsilon}(\mathcal{K}, \Omega, p, m, \varphi),
$$


for every $\epsilon>0$ and some positive constant $C_{\epsilon}$ which depends on $\varphi \in C^{1}(\bar{\Omega})$. Summing up, from (3.7) we deduce

$$
\frac{d}{d t} \int_{\Omega} u^{p}(t) \varphi d x+C \lambda \int_{\Omega} u^{p+m-1}(t) \varphi(x) d x \leq C(\mathcal{K}, \Omega, p, m, \varphi) .
$$

Finally, set $\mu:=(m-1+p) / p>1$ and

$$
Z(t):=\int_{\Omega} u^{p}(t) \varphi d x .
$$

By Jensen's inequality, (3.14) yields the following inequality:

$$
\frac{d}{d t} Z(t)+C_{+}(\lambda)(Z(t))^{\mu} \leq C_{*} .
$$

We can now use Gronwall's inequality (see, e.g., [51, Chapter III, Lemma 5.1]), applied to (3.15), to deduce the desired claim in (3.2). The proof of the proposition is complete.

REMARK 3.1. In the case when $u_{0} \in L^{p}(\Omega), Z(0)=\lim _{t \rightarrow 0^{+}} Z(t)$ is finite, so a similar argument to [51, Chapter III, Lemma 5.1] gives

$$
Z(t) \leq \max \left\{\left\|u_{0}\right\|_{L^{p}(\Omega)},\left(\frac{C_{*}}{C_{+}}\right)^{\frac{1}{\mu}}\right\}, \forall t \geq 0 .
$$

It is worth emphasizing again that the left-hand side of the inequality in (H2) (i.e., $\left.C_{A} y^{m-1} \leq A^{\prime}(y), \forall y \geq 0\right)$ is enough to establish the above assertion. Moreover, the above estimate (3.16) directly implies global well-posedness in the subcritical case for problem (1.1)-(1.3) for as long as $\sigma\left(\Gamma_{D}\right)>0$ (Case (ii)). Unfortunately, we are not able to argue as in the proof of [17, Lemma 10] to obtain the desired $L^{p}$-estimate (similar to the case when $\sigma\left(\Gamma_{D}\right)=0$, see (3.43) below) since we do not know how to get a priori control over the $L^{1}$-norm of $u(t)$ for problem (2.6). We emphasize again that when $\sigma\left(\Gamma_{D}\right)=0$, mass is conserved in both (2.6) and (1.1)-(1.3), so that $\|u(t)\|_{L^{1}(\Omega)} \leq M|\Omega|$ for any $M>0$ such that $\left\langle u_{0}\right\rangle \leq M$.

Next, we establish a crucial result which allows one to deduce a dissipative $L^{\infty}$ estimate uniform with respect to the initial data, and which is necessary for the attractor theory. For this result, we consider both cases $\sigma\left(\Gamma_{D}\right) \geq 0$.

Lemma 3.2. Assume that there exists $\epsilon>0$ and $y_{\epsilon}>0$ such that $A^{\prime}(y) \geq \epsilon$ for all $y \geq y_{\epsilon}$. Let $v$ be a solution of the following degenerate problem:

$$
\partial_{t} v(t)+\nabla \cdot(v(t) \vec{W}(t))=\Delta A(v(t)), \text { in } \Omega \times(0, \infty),
$$

such that $v$ satisfies (1.3) and

$$
\begin{cases}(\nabla A(v)-v \vec{W}) \cdot \vec{n}=0, & \text { on } \Gamma_{N} \times(0, \infty), \\ v=0, & \text { on } \Gamma_{D} \times(0, \infty) .\end{cases}
$$

If

$$
\|\vec{W}(t)\|_{\left(L^{\infty}(\Omega)\right)^{d}} \leq \bar{C}, \text { for all } t>0
$$


then there exists $\mu \sim\left(\tau^{\prime}-\tau\right)>0$, and a positive constant $\mathcal{C}=\mathcal{C}\left(\bar{C}, \mu, \epsilon, y_{\epsilon}\right)$ such that

$$
\sup _{t \geq \tau^{\prime}>0}\|v(t)\|_{L^{\infty}(\Omega)} \leq \mathcal{C} Q\left(1+\sup _{t \geq \tau>0}\|v(t)\|_{L^{1}(\Omega)}\right),
$$

for all $\tau^{\prime}>\tau>0$. The constant $\mathcal{C}=\mathcal{C}(\mu) \sim \mu^{-l}(l>0)$ is bounded if $\mu$ is bounded away from zero.

REMARK 3.2. Lemma 3.2 implies the boundedness of the function $v(t)$ provided that $\vec{W}(t)$ is a bounded vector on $[\tau, \infty)$. It establishes the $L^{1}-L^{\infty}$ smoothing property for the solutions of (3.17)-(3.18). This result is analogous to the result obtained by Kowalczyk [37], but it is more sharp since the function and constant on the right-hand side of (3.19) do not depend on the $L^{\infty}$-norm of the initial data (see [37, Lemma 4.1]). This is very useful if we want to produce uniform estimates with respect to time and the initial data.

Proof. Step 1 (The local relation). First, we recall the following estimate which can be obtained exactly as in [37, Lemma 4.1, (5.1)-(5.4)]. Indeed, setting $v_{m}=(|v|-l)_{+}$, for any $l \geq y_{\epsilon}$, multiplying equation (3.17) by the $p$-th power of $v_{l}$, $p>1$, and integrating by parts using (1.2), it follows, after a suitable re-scaling of the time variable $\bar{t}=\epsilon t$, that

$$
\frac{d}{d \bar{t}} \int_{\Omega} \bar{v}_{l}^{p+1}(\bar{t}) d x+\frac{2 p}{p+1} \int_{\Omega}\left|\nabla \bar{v}_{l}^{\frac{p+1}{2}}(\bar{t})\right|^{2} d x \leq C p(p+1)\left(\int_{\Omega} \bar{v}_{l}^{p+1}(\bar{t}) d x+1\right),
$$

for some constant $C>0$ independent of $p$, but which depends on $l$ and $\epsilon$, and where $\bar{v}_{l}(\bar{t}, x)=v_{l}(\bar{t} / \epsilon, x), \bar{t} \in(0, \epsilon t)$. In fact, $C \sim 1 / \epsilon$ as $\epsilon \rightarrow 0$.

Secondly, set $p_{k}=2^{k}-1, k \geq 0$, and define

$$
\mathcal{Y}_{k}(\bar{t}):=\int_{\Omega} \bar{v}_{l}^{p_{k}+1}(\bar{t}) d x
$$

for all $k \geq 0$. Let $\bar{t}, \mu$ be two positive constants such that $\bar{t}-\mu / p_{k}>0$, and whose values will be chosen later. We claim that there holds

$$
\mathcal{Y}_{k}(\bar{t}) \leq N_{k}(\bar{t}, \mu):=C(\mu, \epsilon)\left(p_{k}\right)^{\gamma}\left(\sup _{s \geq \bar{t}-\mu / p_{k}} \mathcal{Y}_{k-1}(s)+1\right)^{2}, \forall k \geq 1,
$$

where $C, \gamma$ are positive constants independent of $k$. The constant $C=C(\mu, \epsilon)$ is bounded if $\mu$ is bounded away from zero.

We will now prove $(3.22)$ when $2<d$. The case $d \leq 2$ requires only minor modifications by using a suitable Sobolev embedding. The argument we follow is similar, for instance, to [28] (cf. also [27]). For each $k \geq 0$, we define

$$
r_{k}:=\frac{d\left(p_{k}+1\right)-(d-2)\left(1+p_{k}\right)}{d\left(p_{k}+1\right)-(d-2)\left(1+p_{k-1}\right)}, s_{k}:=1-r_{k} .
$$

We aim to estimate the term on the right-hand side of (3.20) in terms of the $L^{1+p_{k-1}}(\Omega)$-norm of $\bar{v}_{m}$. First, the Hölder and Sobolev inequalities (with the equivalent norm of Sobolev spaces in $\left.H^{1}(\Omega) \subset L^{p_{s}}(\Omega), p_{s}=2 d /(d-2)\right)$ yield

$$
\begin{aligned}
\int_{\Omega} \bar{v}_{l}^{p_{k}+1} d x & \leq\left(\int_{\Omega}\left(\bar{v}_{l}\right)^{\frac{\left(p_{k}+1\right) d}{d-2}} d x\right)^{s_{k}}\left(\int_{\Omega}\left(\bar{v}_{l}\right)^{1+p_{k-1}} d x\right)^{r_{k}} \\
& \leq C\left(\int_{\Omega}\left|\nabla \bar{v}_{l}^{\frac{\left(p_{k}+1\right)}{2}}\right|^{2} d x+\int_{\Omega}\left(\bar{v}_{l}\right)^{1+p_{k}} d x\right)^{\bar{s}_{k}} \times\left(\int_{\Omega}\left(\bar{v}_{l}\right)^{1+p_{k-1}} d x\right)^{r_{k}},
\end{aligned}
$$


with $\bar{s}_{k}:=s_{k} d /(d-2) \in(0,1)$. Applying Young's inequality on the right-hand side of (3.23), we get

$$
\int_{\Omega} \bar{v}_{l}^{1+p_{k}} d x \leq \frac{1}{4} \int_{\Omega}\left|\nabla \bar{v}_{l}^{\frac{p_{k}+1}{2}}\right|^{2} d x+Q_{\gamma_{1}}\left(p_{k}\right)\left(\int_{\Omega} \bar{v}_{l}^{p_{k-1}+1} d x\right)^{\theta_{k}}
$$

for some positive constant $\gamma_{1}$ independent of $p_{k}$, and where

$$
\theta_{k}:=\frac{r_{k}}{1-\bar{s}_{k}} \geq 1
$$

(in fact, straightforward computations give $\theta_{k} \equiv 2$ since $p_{k}=2^{k}-1$, for all $k$ ). Note that $Q_{\gamma_{1}}(s) \sim s^{\gamma_{1}}$ as $s \rightarrow \infty$. Inserting estimate (3.24) on the right-hand side of (3.20), we obtain the following inequality:

$$
\frac{d}{d \bar{t}} \mathcal{Y}_{k}(\bar{t})+\frac{3}{4} \int_{\Omega}\left|\nabla \bar{v}_{l}^{\frac{p_{k}+1}{2}}\right|^{2} d x \leq C\left(p_{k}\right)^{\gamma}\left(\mathcal{Y}_{k-1}(\bar{t})+1\right)^{2},
$$

where $C, \gamma$ are positive constants independent of $k$.

We are now ready to prove (3.22) using (3.25). To this end, let $\zeta(s)$ be a positive function $\zeta: \mathbb{R}_{+} \rightarrow[0,1]$ such that $\zeta(s)=0$ for $s \in\left[0, \bar{t}-\mu / p_{k}\right], \zeta(s)=1$ if $s \in[\bar{t},+\infty)$, and $|d \zeta / d s| \leq p_{k} / \mu$, if $s \in\left(\bar{t}-\mu / p_{k}, \bar{t}\right)$. We define $Z_{k}(s)=\zeta(s) \mathcal{Y}_{k}(s)$ and notice that

$$
\frac{d}{d s} Z_{k}(s) \leq \frac{p_{k}}{\mu} \mathcal{Y}_{k}(s)+\zeta(s) \frac{d}{d s} \mathcal{Y}_{k}(s)
$$

Combining this estimate with (3.25), (3.24) and noticing that $Z_{k} \leq \mathcal{Y}_{k}$, we deduce the following estimate for $Z_{k}$ :

$$
\frac{d}{d s} Z_{k}(s)+C(\mu) p_{k} Z_{k}(s) \leq N_{k}(\bar{t}, \mu), \text { for all } s \in\left[\bar{t}-\mu / p_{k},+\infty\right),
$$

for some positive constant $C=C(\mu)$ independent of $k$. Integrating (3.26) with respect to $s$ from $\bar{t}-\mu / p_{k}$ to $\bar{t}$ and taking into account the fact that $Z_{k}\left(\bar{t}-\mu / p_{k}\right)=0$, we obtain that

$$
\mathcal{Y}_{k}(\bar{t})=Z_{k}(\bar{t}) \leq N_{k}(\bar{t}, \mu)\left(1-e^{-C \mu}\right),
$$

which proves the claim (3.22).

Step 2 (The iteration procedure). Let now $\bar{\tau}^{\prime}>\bar{\tau}>0$ be given with $\bar{\tau}>0$ such that

$$
\sup _{s \geq \bar{t}_{1}=\bar{\tau}}\left(\mathcal{Y}_{0}(s)+1\right) \leq \sup _{s \geq \bar{t}_{1}=\bar{\tau}}\left(\|\bar{v}(s)\|_{L^{1}(\Omega)}+1+l|\Omega|\right) \leq C \diamond(l),
$$

and define $\mu=\left(\bar{\tau}^{\prime}-\bar{\tau}\right), \bar{t}_{0}=\bar{\tau}^{\prime}$, and $\bar{t}_{k}=\bar{t}_{k-1}-\mu / p_{k}, k \geq 1$. Using (3.25), we have

$$
\sup _{\bar{t} \geq \bar{t}_{k-1}} \mathcal{Y}_{k}(\bar{t}) \leq C\left(p_{k}\right)^{\gamma}\left(\sup _{s \geq \bar{t}_{k}} \mathcal{Y}_{k-1}(s)+1\right)^{2}, k \geq 1
$$

We can iterate in (3.28) with respect to $k \geq 1$ and obtain that

$$
\begin{aligned}
\sup _{\bar{t} \geq \bar{t}_{k-1}} \mathcal{Y}_{k}(\bar{t}) & \leq\left(C p_{k}^{\gamma}\right)\left(C p_{k-1}^{\gamma}\right)^{2}\left(C p_{k-2}^{\gamma}\right)^{2^{2}} \cdot \ldots \cdot\left(C p_{1}^{\gamma}\right)^{2^{k}}(C \diamond)^{2^{k}} \\
& \leq C^{A_{k}} 2^{B_{k} \gamma}\left(C_{\diamond}\right)^{2^{k}}
\end{aligned}
$$


where

$$
\begin{gathered}
A_{k}:=1+2+2^{2}+\ldots+2^{k}, \\
B_{k}:=k+2(k-1)+2^{2}(k-2)+\ldots+2^{k} .
\end{gathered}
$$

We can easily show that $\left\{A_{k}\right\}$ and $\left\{B_{k}\right\}$ satisfy

$$
A_{k} \leq C\left(2^{k}\right) \text { and } B_{k} \leq C\left(2^{k}\right)
$$

for some positive constant $C$ independent of $k, j$, and $\mu$ (see, e.g., [28]). We can take the $1+p_{k}=2^{k}$-root on both sides of (3.29) and let $k \rightarrow+\infty$. We deduce

$$
\sup _{\bar{t} \geq \bar{t}_{0}=\bar{\tau}^{\prime}}\left\|\bar{v}_{l}(\bar{t})\right\|_{L^{\infty}(\Omega)} \leq \lim _{k \rightarrow+\infty} \sup _{\bar{t} \geq \bar{t}_{0}}\left(\mathcal{Y}_{k}(\bar{t})\right)^{1 /\left(1+p_{k}\right)} \leq C\left(\mu, \epsilon, y_{\epsilon}\right)(C \diamond)
$$

for some positive constant $C$ independent of $\bar{t}, k, \bar{v}_{l}$ and initial data. Next, we notice that, for any $p>1$, in view of $\sqrt[p]{a+b} \leq \sqrt[p]{a}+\sqrt[p]{b}$, there holds

$$
\|\bar{v}(\bar{t})\|_{L^{p}(\Omega)} \leq 2^{\frac{p-1}{p}}\left(\left\|\bar{v}_{l}(\bar{t})\right\|_{L^{p}(\Omega)}+l|\Omega|^{1 / p}\right)
$$

thus, as $p \rightarrow \infty$, we have from (3.33),

$$
\sup _{\bar{t} \geq \bar{\tau}^{\prime}}\|\bar{v}(\bar{t})\|_{L^{\infty}(\Omega)} \leq 2\left(C\left(\mu, \epsilon, y_{\epsilon}\right)(C \diamond)+l\right)
$$

for any $l \geq n_{\epsilon}$. Re-scaling back the time variables $\left(\bar{t}=t \epsilon, \bar{\tau}^{\prime}=\tau^{\prime} \epsilon, \bar{\tau}=\tau \epsilon\right)$ into (3.34) and (3.27), and taking $l=y_{\epsilon}$, we easily obtain the desired inequality (3.19). The proof is finished.

We can now show the following.

THEOREM 3.3. Let $0 \leq u_{0} \in L^{\infty}(\Omega)$ and assume that (H1)-(H3) hold for both cases (i) + (ii). If $\sigma\left(\Gamma_{D}\right)=0$, let $M \geq 0$ be given such that $\left\langle u_{0}\right\rangle \leq M$. Every weak solution of (1.1)-(1.3) is globally Hölder continuous in the cylinders $\left[\tau, \tau^{\prime}\right] \times \bar{\Omega}$, for all $\tau^{\prime}>\tau>0$. In particular, the following estimate holds:

$$
\sup _{t \geq 1}\left(\|A(u)\|_{L^{2}([t, t+1] \times \Omega)}+\|u\|_{C^{\alpha / 2, \alpha}([t, t+1] \times \bar{\Omega})}\right) \leq C, \text { if } \sigma\left(\Gamma_{D}\right)>0 .
$$

Finally, for every bounded subset $B=B(M) \subset L^{1}(\Omega)$, there exists a time $t_{+}=t_{+}(B)>$ 0 such that

$$
\sup _{t \geq t_{+}}\left(\|A(u)\|_{L^{2}([t, t+1] \times \Omega)}+\|u\|_{C^{\alpha / 2, \alpha}([t, t+1] \times \bar{\Omega})}\right) \leq C, \text { if } \sigma\left(\Gamma_{D}\right)=0,
$$

for some $\alpha>0$ and some constant $C>0$ independent of the initial data and time.

Proof. It suffices to prove the estimates (3.35)-(3.36).

Case 1. Consider first $\sigma\left(\Gamma_{D}\right)>0$. In this case, we can take $p>d$ and $p>m+$ $2 d /(d-1)$ as large as we want in Proposition 3.1 so that

$$
\sup _{t \geq \frac{1}{2}}\|\nabla \mathcal{K} * u(t)\|_{L^{p}(\Omega)} \leq C
$$


since $\mathcal{K} \in L^{d /(d-1), \infty}\left(\mathbb{R}^{d}\right)$ (see (3.3)), (3.12). Thus, exploiting first Lemma 2.1-(b), we have for $\vec{V}=\nabla \mathcal{K} * u$

$$
\sup _{t \geq \frac{1}{2}}\|\nabla \vec{V}\|_{L^{p}(\Omega)} \leq C(p) \sup _{t \geq \frac{1}{2}}\|u(t)\|_{L^{p}(\Omega)} \leq C .
$$

By Morrey's inequality (see, e.g., [23]),

$$
\sup _{t \geq \frac{1}{2}}\|\vec{V}\|_{L^{\infty}(\Omega)} \leq C
$$

Invoking now the crucial Lemma 3.2, we deduce on account of (3.2) that

$$
\sup _{t \geq 1}\|u(t)\|_{L^{\infty}(\Omega)} \leq C .
$$

We can now test equation (2.3) with $A(u(t))$, and then integrate over $(t, t+1)$. Setting $\bar{A}(u):=\int_{0}^{u} A(s) d s$ and exploiting (3.40), we obtain, for every $t \geq 1$,

$$
\begin{aligned}
& \int_{\Omega}(\bar{A}(u(t+1))-\bar{A}(u(t))) d x+\int_{t}^{t+1}\|\nabla A(u(s))\|_{L^{2}(\Omega)}^{2} d s \\
= & \int_{t}^{t+1} \int_{\Omega} \nabla A(u(s)) \cdot(u \nabla \mathcal{K} * u) d x d s \\
\leq & \frac{1}{2} \int_{t}^{t+1}\|\nabla A(u(s))\|_{L^{2}(\Omega)}^{2} d s+C .
\end{aligned}
$$

Thus, we easily deduce the first part of (3.35). For every $1<p<\infty$, we now have

$$
\Delta \mathcal{K} * u \in L^{p}(\Omega \times[1, \infty)) \text { and } \vec{V}=\nabla \mathcal{K} * u \in L^{\infty}(\Omega \times[1 / 2, \infty))
$$

since $u$ is bounded. Global Hölder continuity results for (1.1)-(1.2) have been proven for more general classes of degenerate quasilinear equations in divergence form [21, $22,32,33,47]$. Due to $(3.41)$, the second estimate in (3.35) (respectively, (3.36)) is a simple corollary of these results when $m \geq m_{*}$; see, e.g., [20] (cf. also [19, 21, 47]).

Case 2. The case $\sigma\left(\Gamma_{D}\right)=0$ is similar. Following [17, Lemma 10] and Lemma 2.1-(c), we see that for any $m \in\left[1, m_{*}\right]$ such that

$$
\liminf _{z \rightarrow \infty} A^{\prime}(z) z^{1-m}>0
$$

and $\bar{q}=(2-m) /\left(2-m_{*}\right) \geq 1$, with $u_{k}:=(u-k)_{+} \in L^{\bar{q}}(\Omega)$, the following estimate holds (cf. [17, pg. 1703]):

$$
\frac{d}{d t}\left\|u_{k}(t)\right\|_{L^{p}(\Omega)}^{p} \leq-\eta\left\|u_{k}(t)\right\|_{L^{p}(\Omega)}^{p}+C\left(M, \Omega, k, p,\left\|u_{k}(t)\right\|_{L^{\bar{q}}(\Omega)}\right),
$$

for all $t \geq 0$ and some positive constant $\eta>0$. It remains to note that by assumption (H2), condition (3.42) is already satisfied with $m=m_{*}$, so we can choose $\bar{q}=1$ in (3.43). Gronwall's inequality (see, e.g., [51]) applied to (3.43) yields, on account of (2.13), that

$$
\|u(t)\|_{L^{p}(\Omega)}^{p} \leq\left\|\left(u_{0}-k\right)_{+}\right\|_{L^{p}(\Omega)}^{p} e^{-\eta t}+\bar{C}(M, \Omega, k, p),
$$


since $\|u(t)\|_{L^{1}(\Omega)}=|\Omega|\langle u(t)\rangle \leq M|\Omega|$ (mass is conserved). Hence, for every subset $B=B(M) \subset L^{1}(\Omega)$, and setting $R_{*}=\bar{C}(M, \Omega, k, p)+1$, we can easily find a time $t_{+}=$ $t_{+}(M)>0$ such that (3.44) implies

$$
\sup _{t \geq t_{+}}\|u(t)\|_{L^{p}(\Omega)}^{p} \leq R_{*} .
$$

After establishing the bound (3.44), the same estimates (3.37)-(3.39) hold provided that $\nabla \mathcal{K} \in L^{q, \infty}(\Omega)$, for $q>d /(d-1)$ is satisfied. Thus, we can again reach the uniform $L^{\infty}$-estimate (3.40) now on the time intervals $\left[t_{+}, \infty\right)$. This is enough to get (3.36) once more by applying the results in $[20,47]$ (see Case 1 above) and to conclude the proof of the theorem.

Define, for some given $M \geq 0$,

$$
\mathcal{Z}_{D N}:= \begin{cases}\left\{u_{0} \in L^{\infty}(\Omega): u_{0} \geq 0,\left\langle u_{0}\right\rangle \leq M\right\}, & \text { if } \sigma\left(\Gamma_{D}\right)=0 \\ \left\{u_{0} \in L^{\infty}(\Omega): u_{0} \geq 0\right\}, & \text { if } \sigma\left(\Gamma_{D}\right)>0\end{cases}
$$

In the previous section we have proved that system (1.1)-(1.3) generates a continuous semigroup $S(t)$ on the phase space $\mathcal{Z}_{D N}$, endowed with the metric topology of $\left(H_{D}^{1}(\Omega)\right)^{*}$, via

$$
S(t):\left[\mathcal{Z}_{D N}\right]_{*} \rightarrow\left[\mathcal{Z}_{D N}\right]_{*}, S(t) u_{0}=u(t), t \geq 0,
$$

where $u(t)$ is a unique weak solution of (1.1)-(1.3) (see Theorem 2.3). Here $\left[\mathcal{Z}_{D N}\right]_{*}$ denotes the closure of $\mathcal{Z}_{D N}$ in the metric of $\left(H^{1}(\Omega)\right)^{*}$. We devote next our attention to the study of the long-time behavior of trajectories of the semigroup in terms of global attractors. We need to recall the following definition.

DeFinition 3.4. We say that $\mathcal{A} \subset \mathcal{Z}_{D N}$ is the global attractor for the dynamical system $\left(S(t), \mathcal{Z}_{D N}\right)$ if the following conditions are satisfied:

(i) the set $\mathcal{A}$ is a compact subset of the phase space $\mathcal{Z}_{D N}$;

(ii) it is strictly invariant, that is, $S(t) \mathcal{A}=\mathcal{A}$, for all $t \geq 0$;

(iii) for every bounded subset $B \subset \mathcal{Z}_{D N}$,

$$
\operatorname{dist}_{\left[\mathcal{Z}_{D N}\right]_{*}}(S(t) B, \mathcal{A}) \rightarrow 0 \text {, as } t \rightarrow \infty,
$$

that is, $\mathcal{A}$ attracts the images of all bounded subsets of $\mathcal{Z}_{D N}$ as time goes to infinity. Here,

$$
\operatorname{dist}_{\left[\mathcal{Z}_{D N}\right]_{*}}(X, Y)=\sup _{x \in X} \inf _{y \in Y}\|x-y\|_{\left[\mathcal{Z}_{D N}\right]_{*}} .
$$

The first main result of this section states the existence of such an attractor for problem (1.1)-(1.3).

TheOREM 3.5. Let the assumptions of Theorem 3.3 hold. Then the dynamical system $\left(S(t), \mathcal{Z}_{D N}\right)$ associated with problem (1.1)-(1.3) possesses a global attractor $\mathcal{A}_{D N}$ in the phase-space $\mathcal{Z}_{D N}$, which is globally bounded in $C^{\alpha}(\bar{\Omega}), \alpha \in(0,1)$ and has the following structure:

$$
\mathcal{A}_{D N}=\Xi_{\mid t=0}
$$


where $\Xi$ is the set of all bounded solutions of (1.1)-(1.3), defined for all $t \in \mathbb{R}$, such that

$$
\|\Xi\|_{C^{\alpha / 2, \alpha}(\mathbb{R} \times \bar{\Omega})} \leq C
$$

for some positive constant $C$.

Proof. As usual, we must check that $S(t)$ possesses a (pre)compact absorbing set in $\mathcal{Z}_{D N}$, and that it is closed. The first assertion is an immediate corollary of Theorem 3.3. Indeed, for every bounded subset $B \subset \mathcal{Z}_{D N}$, there exists a time $t_{+}=t_{+}(B)>0$, such that $S(t) B \subset \mathcal{X}^{\alpha}$, for all $t \geq t_{+}$, where

$$
\mathcal{X}^{\alpha}:=\left\{u \in C^{\alpha}(\bar{\Omega}):\|u\|_{C^{\alpha}(\bar{\Omega})} \leq C\right\}
$$

( $C$ is the same constant as in (3.35)-(3.36)). Moreover, owing to Lemma 2.4, we also have that $S(t)$ is a closed semigroup in the sense of [46]. Thus, due to the abstract theorem on the attractor's existence [46, Corollary 6$]$, this semigroup possesses a global attractor $\mathcal{A}_{D N}$, bounded in $C^{\alpha}(\bar{\Omega})$. The characterization (3.48) and estimate (3.49) follow from Theorem 3.3 and the same abstract results. Lemma 3.5 is proved.

Remark 3.3. Of course, the global attractor $\mathcal{A}_{D N}$ in Theorem 3.5 depends on $M>0$ since the constant $C>0$ in (3.50) does. Furthermore, the entering time $t_{+}>0$ is even independent of the set $B \subset \mathcal{Z}_{D N}$, when $\sigma\left(\Gamma_{D}\right)>0$, while it only depends on $M>0$ whenever $\sigma\left(\Gamma_{D}\right)=0$.

Finally, we can extend the above result in the critical case $m=m_{*}$ and $\sigma\left(\Gamma_{D}\right)=0$.

THEOREM 3.6. Let assumptions (H1), (H3), and (H2) with $m=m_{*}$ be satisfied, and consider the case when $\Gamma_{D}$ is empty. Let $M_{c}>0$ be the critical mass estimated in [17, Proposition 2 and 3]. Then for every $M<\frac{M_{c}}{|\Omega|}$ such that $\left\langle u_{0}\right\rangle \leq M$, the dynamical system $\left(S(t), \mathcal{Z}_{D N}\right)$ possesses a global attractor $\mathcal{A}_{M_{c}} \subset \mathcal{Z}_{D N}$, bounded in $C^{\alpha}(\bar{\Omega}), \alpha \in$ $(0,1)$.

Proof. As in [17], for every $\int_{\Omega} u_{0} d x=\widetilde{M}<M_{c}$, problem (1.1)-(1.3) is globally well-posed (see, in particular, [17, Theorems 7 and 9]). The assertion in the theorem follows by arguing exactly as in the proof of Theorem 3.3 (Case 2) since (3.42) is still satisfied when $m=m_{*}$.

\section{The non-degenerate case and smooth kernels}

In this section, we are interested in the model proposed in [7] which also takes into account stochastic fluctuations based on a finite number of individuals subject to long range attraction and short range repulsion. In this case, the density $u(t)$ satisfies

$$
\partial_{t} u=\operatorname{div}(\nabla A(u)-u(\nabla \mathcal{K} * u))+\varepsilon \Delta u, \text { in } \Omega \times(0, \infty) .
$$

The additional parameter $\varepsilon>0$ models classical Brownian random dispersal in equation (1.1), which can be seen as the limit of (4.1) as $\varepsilon \rightarrow 0$; see [7]. For the sake of simplicity, we will only consider the no-flux boundary condition for (4.1),

$$
(\nabla(A(u)+\varepsilon u)-u(\nabla \mathcal{K} * u)) \cdot \vec{n}=0, \text { on } \Gamma \times(0, \infty),
$$

and, as before, the initial condition

$$
u_{\mid t=0}=u_{0} \text { in } \Omega .
$$


Thus, everywhere in this section, the boundary $\Gamma_{D}$, where $u=0$, is assumed to be empty (hence, $\Gamma \equiv \partial \Omega$ ).

As in Section 3, we have the following result whose proof is straightforward (see $[17,12])$.

TheOrem 4.1. Let (H1) and (H3) be satisfied and assume

$$
\liminf _{z \rightarrow \infty} A^{\prime}(z) z^{1-m_{*}}>0,
$$

where $m_{*} \geq 1$ is the same critical exponent used in Section 3. If $m_{*}=1$, we further assume that each $M \geq 0$ satisfies $M<\frac{M_{c}}{|\Omega|}$, where $\left\langle u_{0}\right\rangle \leq M$. Then there exists a unique (global) nonnegative solution $u(t)=u_{\varepsilon}(t)$ to problem (4.1)-(4.3), which belongs to (2.2), and, in addition,

$$
u(t) \in L^{2}\left(0, T ; H^{1}(\Omega)\right) .
$$

Each weak solution satisfies

$$
\left\langle\partial_{t} u(t), w\right\rangle_{\left(H^{1}(\Omega)\right)^{*}, H^{1}(\Omega)}+\int_{\Omega} \nabla A_{\varepsilon}(u(t)) \cdot \nabla w-u(t)(\nabla \mathcal{K} * u(t)) \cdot \nabla w d x=0,
$$

for all $w \in H^{1}(\Omega)$, for almost all $t \in[0, T]$, where $A_{\varepsilon}(y):=A(y)+\varepsilon y, \varepsilon>0$.

REMARK 4.1. The weak solution of (4.1)-(4.2) satisfies the energy identity

$$
\mathcal{E}_{\varepsilon}(u(t))+\int_{0}^{t} \int_{\Omega} u(s)\left|\nabla \Phi_{\varepsilon}^{\prime}(u(s))-\nabla \mathcal{K} * u(s)\right|^{2} d x d s=\mathcal{E}_{\varepsilon}(0),
$$

for all $t \geq 0$; see Remark 2.3. The critical mass $M_{c}>0$ in the statement of Theorem 4.1 is estimated in [17, Proposition 2 and 3].

In view of estimate (3.36), the following result can be proven for (4.1)-(4.3).

Lemma 4.2. Let the assumptions of Theorem 4.3 be satisfied. Then, for every $\tau>0$, there exists a constant $C_{M, \varepsilon, \tau}>0$ such that

$$
\sup _{t \geq \tau}\left[\|u(t)\|_{H^{1}(\Omega)}+\left\|\partial_{t} u\right\|_{L^{2}([t, t+1] \times \Omega)}\right] \leq C_{M, \varepsilon, \tau}
$$

Moreover, for any bounded set $\mathcal{B} \subset \mathcal{Z}_{D N}$, there exists a time $t_{*}=t_{*}(\mathcal{B})>0$ such that $S_{\varepsilon}(t) \mathcal{B} \subset H^{1}(\Omega)$, for all $t \geq t_{*}$.

Proof. It suffices to show (4.6) with $\tau=t_{+}+1$ (see (3.44)-(3.45)), where $t_{+}>0$ is the time given in Theorem 3.3 (which still applies for $A_{\varepsilon}(y)=A(y)+\varepsilon y$ ). Set $\bar{A}_{\varepsilon}(y)=\int_{0}^{y} A_{\varepsilon}(s) d s$. Testing equation (4.5) with $w=A_{\varepsilon}(u(t))$, and integrating over $\Omega$, we deduce

$$
\begin{aligned}
& \frac{d}{d t}\left(\bar{A}_{\varepsilon}(u(t)), 1\right)_{L^{1}(\Omega)}+\int_{\Omega}\left|\nabla A_{\varepsilon}(u(t))\right|^{2} d x \\
= & \int_{\Omega} u(t)(\nabla \mathcal{K} * u(t)) \cdot \nabla A_{\varepsilon}(u(t)) d x \\
\leq & \frac{1}{2} \int_{\Omega}\left|\nabla A_{\varepsilon}(u(t))\right|^{2} d x+C\|u(t)\|_{L^{\infty}}^{2}\|\nabla \mathcal{K} * u(t)\|_{L^{2}}^{2} .
\end{aligned}
$$


Integrating this inequality from $t$ to $t+1$, and using the fact that $u$ is bounded according to (3.36), we obtain

$$
\int_{t}^{t+1} \int_{\Omega}\left|\nabla A_{\varepsilon}(u(s))\right|^{2} d x \leq C
$$

for all $t \geq t_{+}(M)$, for some positive constant $C$ independent of time and the initial data.

In order to rigorously prove (4.6), one must proceed as for the problem (2.6). More precisely, recalling that problem (4.1)-(4.3) is uniformly parabolic, one has to employ another regularization scheme in which $A_{\varepsilon}$ is approximated by a sequence of functions $\left(A_{\varepsilon}\right)_{\epsilon} \in C^{\infty}\left(\mathbb{R}_{+}\right)$, the data $u_{\mid t=0}=u_{0 \epsilon} \in C^{\infty}(\Omega) \cap C^{1}(\bar{\Omega})$ is such that $u_{0 \epsilon} \rightarrow u_{0}$ in the $L^{\infty}$-metric, and $\mathcal{K}$ is replaced by a sequence of smooth kernels $\mathcal{K}_{\epsilon} \in C^{\infty}$ such that $\mathcal{K}_{\epsilon} \rightarrow$ $\mathcal{K}$ in $W_{\text {loc }}^{1,1}\left(\mathbb{R}^{d}\right)$, as $\epsilon \rightarrow 0$. The procedure ensures that the approximate solutions $\left(u_{\varepsilon}\right)_{\epsilon}$ are smooth enough so that all the computations below can be performed rigorously. Thus, in what follows we shall again proceed formally (it will be easy to see that all the constants in the estimates are independent of $\left.\epsilon \rightarrow 0^{+}\right)$. To this end, testing equation (4.5) with $w=\partial_{t}\left(A_{\varepsilon} u(t)\right)$, and using the fact that $A_{\varepsilon}^{\prime}(y) \geq \varepsilon>0$, we deduce

$$
2 \varepsilon \int_{\Omega}\left|\partial_{t} u\right|^{2} d x+\frac{d}{d t} \int_{\Omega}\left|\nabla A_{\varepsilon}(u)\right|^{2} d x=2 \int_{\Omega} u(\nabla \mathcal{K} * u) \cdot \nabla \partial_{t} A_{\varepsilon}(u) d x .
$$

Since

$$
\begin{gathered}
\int_{\Omega} u(\nabla \mathcal{K} * u) \cdot \nabla \partial_{t} A_{\varepsilon}(u) d x=\frac{d}{d t} \int_{\Omega} u(\nabla \mathcal{K} * u) \cdot \nabla A_{\varepsilon}(u) d x-\int_{\Omega} \partial_{t} u(\nabla \mathcal{K} * u) \cdot \nabla A_{\varepsilon}(u) d x \\
-\int_{\Omega} u\left(\nabla \mathcal{K} * \partial_{t} u\right) \cdot \nabla A_{\varepsilon}(u) d x
\end{gathered}
$$

relation (4.9) and the basic Hölder and Young inequalities imply

$$
\begin{aligned}
& \varepsilon \int_{\Omega}\left|\partial_{t} u\right|^{2} d x+\frac{d}{d t} \int_{\Omega}\left|\nabla A_{\varepsilon}(u)\right|^{2} d x \\
\leq & \frac{d}{d t} \int_{\Omega} 2 u(\nabla \mathcal{K} * u) \cdot \nabla A_{\varepsilon}(u) d x+c \int_{\Omega}\left|\nabla A_{\varepsilon}(u)\right|^{2} d x,
\end{aligned}
$$

for some positive constant $c>0$ independent of time and the initial data. Next, we multiply (4.10) by $e^{c(t-s)}$ for $s \in(t, t+1)$ to obtain

$$
\frac{d}{d s} e^{c(t-s)} \int_{\Omega}\left|\nabla A_{\varepsilon}(u)\right|^{2} d x \leq e^{c(t-s)} \frac{d}{d s} \int_{\Omega} 2 u(\nabla \mathcal{K} * u) \cdot \nabla A_{\varepsilon}(u) d x .
$$

Integrating (4.11) between $s$ and $t+1$ gives

$$
\begin{aligned}
& e^{-c} \int_{\Omega}\left|\nabla A_{\varepsilon}(u(t+1))\right|^{2} d x-e^{c(t-s)} \int_{\Omega}\left|\nabla A_{\varepsilon}(u(s))\right|^{2} d x \\
\leq & \int_{s}^{t+1} e^{c(t-\tau)} \frac{d}{d \tau} \int_{\Omega} 2 u(\tau)(\nabla \mathcal{K} * u(\tau)) \cdot \nabla A_{\varepsilon}(u(\tau)) d x d \tau .
\end{aligned}
$$


Notice that we can split the integral on the right-hand side of (4.12) as follows:

$$
\begin{aligned}
& \int_{s}^{t+1} e^{c(t-\tau)} \frac{d}{d \tau} \int_{\Omega} 2 u(\tau)(\nabla \mathcal{K} * u(\tau)) \cdot \nabla A_{\varepsilon}(u(\tau)) d x d \tau \\
= & \left.e^{c(t-\tau)} \int_{\Omega} 2 u(\tau)(\nabla \mathcal{K} * u(\tau)) \cdot \nabla A_{\varepsilon}(u(\tau)) d x\right|_{s} ^{t+1} \\
& \quad-\int_{s}^{t+1}(-c) e^{c(t-\tau)} \int_{\Omega} 2 u(\tau)(\nabla \mathcal{K} * u(\tau)) \cdot \nabla A_{\varepsilon}(u(\tau)) d x d \tau \\
= & : I_{6}+I_{7} .
\end{aligned}
$$

Next,

$$
\begin{array}{r}
I_{6}=\left.e^{c(t-\tau)} \int_{\Omega} 2 u(\tau)(\nabla \mathcal{K} * u(\tau)) \cdot \nabla A_{\varepsilon}(u(\tau)) d x\right|_{s} ^{t+1} \\
=e^{-c} \int_{\Omega} 2 u(t+1)(\nabla \mathcal{K} * u(t+1)) \cdot \nabla A_{\varepsilon}(u(t+1)) d x \\
\quad-e^{c(t-s)} \int_{\Omega} 2 u(s)(\nabla \mathcal{K} * u(s)) \cdot \nabla A_{\varepsilon}(u(s)) d x,
\end{array}
$$

which can be further bounded, exploiting the standard Hölder and Young inequalities, by

$$
\begin{gathered}
I_{6} \leq \frac{e^{-c}}{2} \int_{\Omega}\left|\nabla A_{\varepsilon}(u(t+1))\right|^{2} d x+C\|u(t+1)\|_{L^{\infty}(\Omega)}^{2} \\
+\int_{\Omega}\left|\nabla A_{\varepsilon}(u(s))\right|^{2} d x+C\|u(s)\|_{L^{\infty}(\Omega)}^{2} .
\end{gathered}
$$

Moreover, we have

$$
\begin{aligned}
I_{7} & =\int_{s}^{t+1} c e^{c(t-\tau)} \int_{\Omega} 2 u(\tau)(\nabla \mathcal{K} * u(\tau)) \cdot \nabla A_{\varepsilon}(u(\tau)) d x d \tau \\
& \leq C \int_{s}^{t+1}\left(\int_{\Omega}|u(\tau)|^{2} d x+\int_{\Omega}\left|\nabla A_{\varepsilon}(u(\tau))\right|^{2} d x\right) d \tau .
\end{aligned}
$$

Thus, on account of (4.13)-(4.14) and estimate (4.19), inequality (4.12) becomes

$$
\begin{aligned}
& \quad e^{-c} \int_{\Omega}\left|\nabla A_{\varepsilon}(u(t+1))\right|^{2} d x-e^{c(t-s)} \int_{\Omega}\left|\nabla A_{\varepsilon}(u(s))\right|^{2} d x \\
& \leq \frac{e^{-c}}{2} \int_{\Omega}\left|\nabla A_{\varepsilon}(u(t+1))\right|^{2} d x+\int_{\Omega}\left|\nabla A_{\varepsilon}(u(s))\right|^{2} d x \\
& \quad+C \int_{s}^{t+1}\left(\int_{\Omega}|u(\tau)|^{2} d x+\int_{\Omega}\left|\nabla A_{\varepsilon}(u(\tau))\right|^{2} d x\right) d \tau+C,
\end{aligned}
$$

for all $t \geq t_{+}$. Therefore,

$$
\begin{aligned}
& \quad \frac{e^{-c}}{2} \int_{\Omega}\left|\nabla A_{\varepsilon}(u(t+1))\right|^{2} d x \\
& \leq e^{c(t-s)} \int_{\Omega}\left|\nabla A_{\varepsilon}(u(s))\right|^{2} d x+\int_{\Omega}\left|\nabla A_{\varepsilon}(u(s))\right|^{2} d x \\
& \quad+C \int_{s}^{t+1}\left(\int_{\Omega}|u(\tau)|^{2} d x+\int_{\Omega}\left|\nabla A_{\varepsilon}(u(\tau))\right|^{2} d x\right) d \tau+C .
\end{aligned}
$$


Integrating this inequality from $t$ to $t+1$ with respect to $s$, and recalling (4.19), we obtain

$$
\begin{aligned}
& \frac{e^{-c}}{2} \int_{\Omega}\left|\nabla A_{\varepsilon}(u(t+1))\right|^{2} d x \\
\leq & \int_{t}^{t+1} e^{c(t-s)} \int_{\Omega}\left|\nabla A_{\varepsilon}(u(s))\right|^{2} d x d s+\int_{t}^{t+1} \int_{\Omega}\left|\nabla A_{\varepsilon}(u(s))\right|^{2} d x \\
& \quad+C \int_{t}^{t+1} \int_{s}^{t+1}\left(\int_{\Omega}|u(\tau)|^{2} d x+\int_{\Omega}\left|\nabla A_{\varepsilon}(u(\tau))\right|^{2} d x\right) d \tau+C \\
\leq & C \int_{t}^{t+1} \int_{\Omega}\left|\nabla A_{\varepsilon}(u(s))\right|^{2} d x+C \int_{t}^{t+1}(\tau-t) \int_{\Omega}\left|\nabla A_{\varepsilon}(u(\tau))\right|^{2} d x d \tau+C .
\end{aligned}
$$

By virtue of (4.8), (4.16) yields

$$
\int_{\Omega}\left|\nabla A_{\varepsilon}(u(t+1))\right|^{2} d x \leq C, \forall t \geq t_{+} .
$$

Since $A_{\varepsilon}^{\prime}(y) \geq \varepsilon$, for all $y$, and integrating (4.10) over $(t, t+1)$ once more, (4.17) entails the desired estimate (4.6). The proof is finished.

As in Section 3, we can prove the following result for (4.1)-(4.3).

TheOREM 4.3. Let the assumptions of Theorem 4.1 be satisfied. The dynamical system $\left(S_{\varepsilon}(t), \mathcal{Z}_{D N}\right)\left(\left\{S_{\varepsilon}(t)\right\}_{t \geq 0}\right.$ defined as in (3.46)) possesses a global attractor $\mathcal{A}=\mathcal{A}_{\varepsilon, M}$ in the sense of Definition 3.4, such that $\mathcal{A}$ is globally bounded in $C^{\alpha}(\bar{\Omega}) \cap$ $H^{1}(\Omega)$, for some $\alpha \in(0,1)$.

Proof. As in the proof of Theorem 3.5, we must check that $S_{\varepsilon}(t)$ is closed and that it admits a compact absorbing set in $\mathcal{Z}_{D N}$.

Step 1 (Closedness of $S_{\varepsilon}$ ). The proof of this step is essentially the same as in Lemma 2.4, where everywhere in the estimates we must replace $A$ by the function $A_{\varepsilon}$. This only affects the estimate for $(2.20)$, which now reads

$$
\begin{aligned}
& I_{1}=-\int_{\Omega}\left(A_{\varepsilon}\left(u_{1}\right)-A_{\varepsilon}\left(u_{2}\right)\right)\left(u_{1}-u_{2}\right) d x+M_{12} \int_{\Omega} A_{\varepsilon}\left(u_{1}\right)-A_{\varepsilon}\left(u_{2}\right) d x \\
& =-\varepsilon\left\|u_{1}-u_{2}\right\|_{L^{2}(\Omega)}^{2}-\int_{\Omega}\left(A\left(u_{1}\right)-A\left(u_{2}\right)\right)\left(u_{1}-u_{2}\right) d x \\
& \quad \quad+\varepsilon\left(M_{12}\right)^{2}+M_{12} \int_{\Omega} A\left(u_{1}\right)-A\left(u_{2}\right) d x \\
& \quad \leq-\varepsilon\left\|u_{1}-u_{2}\right\|_{L^{2}(\Omega)}^{2}+C(1+\varepsilon)\left(M_{1}-M_{2}\right)^{2} .
\end{aligned}
$$

Thus, the same inequality in (2.15) is valid for any two weak solutions $u_{1}(t), u_{2}(t)$ of problem (4.1)-(4.3) corresponding to the initial data $u_{10}, u_{20}$.

Step 2 (Smoothing effect). This step requires only minor modifications in the proof of Theorem 3.3. Indeed, Lemma 3.2 also applies to the function $A_{\varepsilon}$ and the results in, e.g., [20, Corollary 4.2] can be still applied to obtain global Hölder continuity of the weak solutions. In particular, each weak solution of (4.1)-(4.3) satisfies the $L^{1}$ $C^{\alpha} \cap H^{1}$ smoothing property, and there exists a time $t_{+}=t_{+}(M)>0$ such that

$$
\sup _{t \geq t_{+}}\|u\|_{C^{\alpha / 2, \alpha}([t, t+1] \times \bar{\Omega}) \cap L^{\infty}\left(\left[t_{+} ; \infty\right) ; H^{1}(\Omega)\right)} \leq C_{M},
$$


for some positive constant $C_{M}$, independent of time and the initial data. Hence, a compact in $\mathcal{Z}_{D N}$ absorbing set like in (3.50) can be sought, which is enough to apply [46, Corollary 6] once again. The proof is finished.

Next, taking advantage of the asymptotic smoothness of $S_{\varepsilon}(t)$ we can also show that (4.1)-(4.3) has a gradient structure. To this end, we define the $\omega$-limit set of a trajectory $u(t)$ of (4.1)-(4.3), starting from

$$
u_{0} \in \mathcal{Z}_{D N}=\left\{u_{0} \in L^{\infty}(\Omega): u_{0} \geq 0,\left\langle u_{0}\right\rangle \leq M\right\},
$$

as follows:

$$
\omega\left(u_{0}\right)=\left\{u_{*} \in \mathcal{Z}_{D N}: \exists t_{n} \rightarrow \infty \text { such that } \lim _{n \rightarrow \infty}\left\|u\left(t_{n}\right)-u_{*}\right\|_{L^{\infty}(\Omega)}=0\right\} .
$$

We prove that $\omega\left(u_{0}\right)$ consists of stationary solutions, satisfying the system

$$
\left\{\begin{array}{l}
\operatorname{div}\left(u_{*} \nabla\left(\Phi_{\varepsilon}^{\prime}\left(u_{*}\right)-\mathcal{K} * u_{*}\right)\right)=0, \text { in } \Omega, \\
u_{*} \nabla\left(\Phi_{\varepsilon}^{\prime}\left(u_{*}\right)-\mathcal{K} * u_{*}\right) \cdot \vec{n}=0, \quad \text { on } \Gamma, \\
\left\langle u_{*}\right\rangle:=\frac{1}{|\Omega|} \int_{\Omega} u_{*}(x) d s=\left\langle u_{0}\right\rangle,
\end{array}\right.
$$

where we recall that $\Phi_{\varepsilon}^{\prime \prime}(y)=A_{\varepsilon}^{\prime}(y) / y$ such that $\Phi_{\varepsilon}^{\prime}(1)=\Phi_{\varepsilon}(0)=0$ (see Remark 2.3).

The following proposition justifies to call

$$
\mathcal{E}_{\varepsilon}(u(t))=\int_{\Omega} \Phi_{\varepsilon}(u(t)) d x-\frac{1}{2} \int_{\Omega} \int_{\Omega} u(x, t) \mathcal{K}(x-y) u(y, t) d x d y
$$

an energy functional for (4.1)-(4.3).

Proposition 4.4. Let $u(t)=S_{\varepsilon}(t) u_{0}, u_{0} \in \mathcal{Z}_{D N}$, be the (unique) global solution of the non-degenerate aggregation equation (4.1)-(4.3). Then the following assertions are true:

(i) The function $\mathcal{E}_{\varepsilon}(u(t))$ is differentiable a.e. on $[\tau, \infty)$, for every $\tau>0$, and

$$
\frac{d}{d t} \mathcal{E}_{\varepsilon}(u(t))=-\int_{\Omega} u(t)\left|\nabla \Phi_{\varepsilon}^{\prime}(u(t))-\nabla \mathcal{K} * u(t)\right|^{2} d x
$$

for a.e. $t>0$.

(ii) The function $\mathcal{E}_{\varepsilon}(u(t))$ is nonincreasing, and there exists a positive constant $C_{*}$, depending only on $\mathcal{K}, \Omega, \varepsilon$, and $\|u\|_{L^{\infty}(\Omega)}$ such that

$$
\mathcal{E}_{\varepsilon}(u(t)) \geq-C_{*},
$$

for a.e. $t \geq 0$. Moreover, $\lim _{t \rightarrow \infty} \mathcal{E}_{\varepsilon}(u(t))=\inf _{t>0} \mathcal{E}_{\varepsilon}(u(t))=\mathcal{E}_{\varepsilon, \infty} \in \mathbb{R}$ exists.

(iii) For any $u_{0} \in \mathcal{Z}_{D N}, \omega\left(u_{0}\right)$ is a compact, connected invariant set, and every $u_{*} \in \omega\left(u_{0}\right)$ is a solution of the stationary problem (4.21).

(iv) Every $u_{*} \in \omega\left(u_{0}\right)$ is a critical point of $\mathcal{E}_{\varepsilon}(u(t))$ in (4.22), i.e, $\mathcal{E}_{\varepsilon}^{\prime}\left(u_{*}\right)=0$.

Proof. First, note that by the assumption (H2) and (4.4), we have $\Phi(y) \sim$ $A(y) \sim y^{m}$ with $m \geq m_{*}$, and by definition, $\Phi_{\varepsilon}(y)=\Phi(y)+\varepsilon(y \ln (y)-y)$, for every $\varepsilon>0$. The first part of assertion (i) follows from the fact that $\Phi_{\varepsilon} \in C^{1}$ and the regularity of the bounded solution $u(t)$ on the intervals $[\tau, \infty)$, for every $\tau>0$ (indeed, $u \in$ 
$\left.C^{\alpha / 2}\left(\tau, \infty ; C^{\alpha}(\bar{\Omega})\right)\right)$. The second part is a consequence of Remark 4.1. By (4.23), $\mathcal{E}_{\varepsilon}(u(t))$ is nonincreasing on $\mathcal{Z}_{D N}$. The elementary inequality $y \ln (y)-y \geq-1$, for all $y \geq 0$, and the assumption (H2) on $A$, yields on $\mathcal{Z}_{D N}$ that $\Phi_{\varepsilon}(u(t)) \geq-C_{\varepsilon}$, for some constant $C_{\varepsilon}>0$ depending only on the physical parameters of the problem and the $L^{\infty}$-bound of $u$. Rewriting the energy $\mathcal{E}_{\varepsilon}(t)$ in the equivalent form

$$
\begin{gathered}
\mathcal{E}_{\varepsilon}(u(t))=\int_{\Omega} \Phi_{\varepsilon}(u(t)) d x+\frac{1}{4} \iint_{\Omega \times \Omega} \mathcal{K}(x-y)(u(x, t)-u(y, t))^{2} d x d y \\
-\frac{1}{2} \int_{\Omega} a(x)(u(x, t))^{2} d x,
\end{gathered}
$$

where

$$
a(x)=\int_{\Omega} \mathcal{K}(x-y) d y \in C(\bar{\Omega})
$$

the second part of assertion (ii) is also immediate. First, by Theorem 4.1, problem (4.1)-(4.3) defines a nonlinear $C_{0}$-semigroup $S_{\varepsilon}(t): \mathcal{Z}_{D N} \rightarrow \mathcal{Z}_{D N}, u(t)=S_{\varepsilon}(t) u_{0}$ with $u_{0} \in \mathcal{Z}_{D N}$. Second, by the results of theorems 3.3 and 4.2, we know that for any $u_{0} \in \mathcal{Z}_{D N}$ there is $t_{+}>0$ such that $\cup_{t \geq t_{+}} S_{\varepsilon}(t) u_{0}$ is bounded in $H^{1}(\Omega) \cap C^{\alpha}(\bar{\Omega})$, and hence relatively compact in $\mathcal{Z}_{D N}$ (when endowed with the metric topology of $L^{\infty}(\Omega)$ ). Third, it can be seen from (i) that the function $\mathcal{E}_{\varepsilon}(u(t)): \mathcal{F}_{D N} \rightarrow \mathbb{R}$ is a Lyapunov function on $\mathcal{F}_{D N} \subset \mathcal{Z}_{D N}$, for any (positively invariant) subset $\mathcal{F}_{D N} \subset H^{1}(\Omega) \cap C^{\alpha}(\bar{\Omega})$. In particular, by Theorem 4.3 we can take $\mathcal{F}_{D N}=\mathcal{A}_{\varepsilon, M}$, where $\mathcal{A}_{\varepsilon, M}$ is the global attractor for $\left(S_{\varepsilon}(t), \mathcal{Z}_{D N}\right)$. Moreover, $\mathcal{E}_{\varepsilon}$ satisfies: if for $t>0, \mathcal{E}_{\varepsilon}\left(S_{\varepsilon}(t) u_{*}\right)=\mathcal{E}_{\varepsilon}\left(u_{*}\right)$, then $u_{*}$ is an equilibrium point of $S_{\varepsilon}(t)$. In conclusion, by [48, Chapter 10, Definition 10.1], $\left(S_{\varepsilon}(t), \mathcal{A}_{\varepsilon, M}\right)$ is a gradient system. Thus, by [48, Propositions 10.3 and 10.12$]$ we immediately conclude (iii), i.e., $\omega\left(u_{0}\right)$ is a nonempty, compact, connected invariant set, and $\omega\left(u_{0}\right)$ consists only of stationary solutions. The final part of (ii), $\inf _{t>0} \mathcal{E}_{\varepsilon}(u(t))=$ $\mathcal{E}_{\varepsilon}\left(u_{*}\right)=\mathcal{E}_{\varepsilon, \infty}$ is satisfied owing to (4.23), (4.24), and (iii).

Finally, for (iv) we observe that if $u_{*} \in \omega\left(u_{0}\right)$ is a solution of (4.21), then for any $\psi \in H^{1}(\Omega) \cap L^{\infty}(\Omega)$ it follows from (4.21) that

$$
\begin{aligned}
0 & =\int_{\Omega} \operatorname{div}\left(u_{*} \nabla\left(\Phi_{\varepsilon}^{\prime}\left(u_{*}\right)-\mathcal{K} * u_{*}\right)\right) \psi d x-\int_{\Gamma} u_{*} \nabla\left(\Phi_{\varepsilon}^{\prime}\left(u_{*}\right)-\mathcal{K} * u_{*}\right) \cdot \vec{n} \psi d \sigma \\
& =-\int_{\Omega}\left(\nabla A_{\varepsilon}\left(u_{*}\right)-u_{*} \nabla \mathcal{K} * u_{*}\right) \cdot \nabla \psi d x,
\end{aligned}
$$

which, by straightforward computations, is just the following:

$$
\frac{d}{d \delta} \mathcal{E}_{\varepsilon}\left(u_{*}+\delta \psi\right)_{\mid \delta=0}=0,
$$

i.e., $u_{*}$ is also a critical point of $\mathcal{E}_{\varepsilon}$ in $\mathcal{Z}_{D N}$. In fact, we easily see that the statements (iii) and (iv) are equivalent to each other. The proof is complete.

Next, we show that $\omega\left(u_{0}\right)$ has a positive bound from below depending only on the physical parameters of the problem and $u_{0}$.

Proposition 4.5. Let $u(t)=S_{\varepsilon}(t) u_{0}, u_{0} \in \mathcal{Z}_{D N}$, be the unique solution of (4.1)(4.3) such that $\left\langle u_{0}\right\rangle>0$. Suppose that $u_{*} \in \omega\left(u_{0}\right)$. Then, there exists a constant $\underline{u}>0$, depending only on $u_{0}, \Omega, \mathcal{K}$, and $\varepsilon>0$, such that $u_{*}(x) \geq \underline{u}>0$, for all $x \in \Omega$. 
Proof. The proof follows a similar argument used in [26, Section 2], [54, Proposition 3.3]. Indeed, owing to the homogeneous Neumann boundary condition of (4.21), the first equation of (4.21) also reads

$$
\int_{\Omega} u_{*} \nabla\left(\Phi_{\varepsilon}^{\prime}\left(u_{*}\right)-\mathcal{K} * u_{*}\right) \cdot \nabla \psi d x=0,
$$

for any $\psi \in C^{1}(\bar{\Omega})$, whence $\nabla\left(\Phi_{\varepsilon}^{\prime}\left(u_{*}\right)-\mathcal{K} * u_{*}\right)=0$ in each connected component of the open set where $x \in \Omega$ such that $u_{*}(x)>0$. Therefore, $\Phi_{\varepsilon}^{\prime}\left(u_{*}\right)-\mathcal{K} * u_{*}$ is constant on each such connected component. If $\left\langle u_{0}\right\rangle>0$, by the last equation of (4.21), there exists $x_{0} \in \Omega$ such that $u_{*}\left(x_{0}\right)>0$. The previous statement implies that there exists $\gamma \in \mathbb{R}$ such that

$$
\gamma=\Phi_{\varepsilon}^{\prime}\left(u_{*}(x)\right)-\left(\mathcal{K} * u_{*}\right)(x)=\Phi^{\prime}\left(u_{*}(x)\right)+\varepsilon \ln \left(u_{*}(x)\right)-\left(\mathcal{K} * u_{*}\right)(x),
$$

for $x \in \Omega\left(x_{0}\right)$, which is the same connected component of $\left\{x \in \Omega: u_{*}(x)>0\right\}$ as $x_{0}$. Since $u_{*} \in \omega\left(u_{0}\right)$ is bounded and $\mathcal{K} \in W_{\text {loc }}^{1,1}\left(\mathbb{R}^{d}\right)$, we observe from (4.25) that $u_{*}(x)$ satisfies the inequality

$$
\left.u_{*}(x) \geq e^{\frac{1}{\varepsilon}\left(\gamma-\left\|\mathcal{K} * u_{*}\right\|_{L}(\Omega)\right.}-\left\|\Phi^{\prime}\left(u_{*}(x)\right)\right\|_{L^{\infty}(\Omega)}\right)>0,
$$

for all $x \in \Omega\left(x_{0}\right)$. In particular, this yields that $\Omega\left(x_{0}\right)=\Omega$ and the claim is proved. $\square$

REMARK 4.2. Some results on properties of the steady states for the aggregation equation with nonlinear diffusion (1.1) in the case $\Omega=\mathbb{R}^{d}$ can be found $[5,8,9]$.

As a result of the proof of Proposition 4.4, we can now conclude the following

THEOREM 4.6. Let the assumptions of Theorem 4.3 be satisfied. The global attractor $\mathcal{A}=\mathcal{A}_{\varepsilon, M}$ of problem (4.1)-(4.3) consists entirely of unstable manifolds of the equilibria, which are bounded solutions of (4.21).

Under an additional assumption on the kernel which ensures that $\mathcal{K}$ is reasonably smooth at the origin, we can show that $\mathcal{A}_{\varepsilon, M}$ is also finite dimensional.

Theorem 4.7. Let the assumptions of Theorem 4.1 be satisfied. Assume

$$
\left(D^{2} \mathcal{K}\right) 1_{B_{1}(0)} \in L^{1}\left(\mathbb{R}^{d}\right), d \geq 2 .
$$

The global attractor $\mathcal{A}_{\varepsilon, M}$ of (4.1)-(4.3) has finite fractal dimension:

$$
\operatorname{dim}_{F}\left(\mathcal{A}_{\varepsilon, M}, H^{1-}(\Omega) \cap L^{p}(\Omega)\right) \leq C_{\varepsilon, M}<\infty,
$$

for any $1<p<\infty$.

REMARK 4.3. Recall that fractal dimension of a compact set $\mathcal{Y} \operatorname{dim}_{F}(\mathcal{Y}, X)$ is defined as

$$
\operatorname{dim}_{F}(\mathcal{Y}, X)=\lim _{\delta \rightarrow 0^{+}} \frac{\log _{2} N_{\delta}(\mathcal{Y}, X)}{\log _{2}(1 / \delta)},
$$

where $N_{\delta}(\mathcal{Y}, X)$ is the minimal number of balls $B_{\delta}$ that can be used to cover the compact set $\mathcal{Y}$ in the metric of $X$. 
The statement of Theorem 4.7 is in fact a consequence of a much stronger result which states that (4.1)-(4.3) admits an exponential attractor $\mathcal{M}_{\varepsilon, M}$ provided that (4.26) is also satisfied. The precise statement is given by the following.

TheOREm 4.8. Let the assumptions of Theorem 4.7 be satisfied. Assume (4.26). For every fixed $\varepsilon>0$, for the dynamical system $\left(S_{\varepsilon}(t), \mathcal{Z}_{D N}\right)$ there exists an exponential attractor $\mathcal{M}=\mathcal{M}_{\varepsilon, M}$, bounded in $C^{\alpha}(\bar{\Omega})$, which satisfies the following properties:

(i) Semi-invariance: $S_{\varepsilon}(t) \mathcal{M} \subset \mathcal{M}$, for every $t \geq 0$.

(ii) Exponential attraction: For every bounded subset $\mathcal{B} \subset \mathcal{Z}_{D N}$,

$$
\operatorname{dist}_{H^{1-}(\Omega) \cap L^{p}(\Omega)}\left(S_{\varepsilon}(t) \mathcal{B}, \mathcal{M}\right) \leq C e^{-\kappa t}, \quad \forall t \geq 0,
$$

for some positive constants $C=C(\varepsilon, M)$ and $\kappa$, for any $1<p<\infty$.

(iii) Finite dimensionality:

$$
\operatorname{dim}_{F}\left(\mathcal{M}, H^{1-}(\Omega) \cap L^{p}(\Omega)\right) \leq C_{\varepsilon, M}<\infty,
$$

for any $1<p<\infty$. The constants $C, C_{\varepsilon, M}, \kappa$ can be computed explicitly in terms of the physical parameters of the problem.

Here, and everywhere else, we denote $H^{1-}(\Omega):=H^{1-\delta}(\Omega)$, for any $\delta \in(0,1]$. Since the global attractor $\mathcal{A}_{\varepsilon, M}$ is always contained in $\mathcal{M}_{\varepsilon, M}$, the above theorem immediately implies that the fractal dimension of the global attractor $\mathcal{A}_{\varepsilon, M}$ is also finite. It is worth recalling that, in the global attractors theory, it is usually extremely difficult (if not impossible) to estimate and to express the rate of convergence in (3.47) in terms of the physical parameters of the system considered. This constitutes the main drawback of the theory. Simple examples show that the rate of convergence in (3.47) can be arbitrarily slow and non-uniform with respect to the parameters of the system considered. As a consequence, the global attractor becomes sensitive to small perturbations and, in some sense, cannot even be observed in experiments. The concept of exponential attractor overcomes this difficulty (see, e.g., the survey article [45]). Indeed, in contrast to the global attractors theory, the constants $C, C_{\varepsilon, M}, \kappa$ in (4.27)-(4.28) can be explicitly found in terms of the physical parameters.

We report for the reader's convenience the following abstract result on the existence of exponential attractors [24, Proposition 4.1] (cf. also [28, Proposition 2.17]) which will be used in the proof of Theorem 4.8.

Proposition 4.9. Let $\mathcal{H}, \mathcal{V}$, and $\mathcal{V}_{1}$ be Banach spaces such that the embedding $\mathcal{V}_{1} \subset \mathcal{V}$ is compact. Let $B$ be a closed bounded subset of $\mathcal{H}$ and let $\mathbb{S}: B \rightarrow B$ be a map. Assume also that there exists a uniformly Lipschitz continuous map $\mathbb{T}: B \rightarrow \mathcal{V}_{1}$, i.e.,

$$
\left\|\mathbb{T} b_{1}-\mathbb{T} b_{2}\right\|_{\mathcal{V}_{1}} \leq L\left\|b_{1}-b_{2}\right\|_{\mathcal{H}}, \quad \forall b_{1}, b_{2} \in B
$$

for some $L \geq 0$, such that

$$
\left\|\mathbb{S} b_{1}-\mathbb{S} b_{2}\right\|_{\mathcal{H}} \leq \theta\left\|b_{1}-b_{2}\right\|_{\mathcal{H}}+K\left\|\mathbb{T} b_{1}-\mathbb{T} b_{2}\right\|_{\mathcal{V}}, \quad \forall b_{1}, b_{2} \in B
$$

for some $\theta<\frac{1}{2}$ and $K \geq 0$. Then there exists a (discrete) exponential attractor $\mathcal{M}_{d} \subset B$ of the semigroup $\left\{\mathbb{S}(n):=\mathbb{S}^{n}, n \in Z+\right\}$ with discrete time in the phase space $\mathcal{H}$.

We delay the proof of Theorem 4.8 until the end of the section. The idea is to verify (4.29)-(4.30) for a suitable choice of maps. We begin by showing that the semigroup $S_{\varepsilon}(t)$ is strongly (Lipschitz) continuous with respect to the $\left(H^{1}(\Omega)\right)^{*}$ metric. 
Proposition 4.10. Let $u_{i}, i=1,2$, be a pair of weak solutions according to the assumptions of Theorem 4.7. Then the following estimate holds:

$$
\begin{aligned}
& \left\|u_{1}(t)-u_{2}(t)\right\|_{\left(H^{1}(\Omega)\right)^{*}}^{2}+\varepsilon \int_{0}^{t}\left\|u_{1}(s)-u_{2}(s)\right\|_{L^{2}(\Omega)}^{2} d s \\
\leq & \left\|u_{1}(0)-u_{2}(0)\right\|_{\left(H^{1}(\Omega)\right)^{*}}^{2} e^{\kappa t},
\end{aligned}
$$

for all $t \geq 0$, for some positive constants $\kappa, C$ which depend on $\varepsilon>0$ and $\mathcal{K}$ but are independent of $u_{i}(0)$.

Proof. Following Lemma 2.4, we have that $u:=u_{1}-u_{2}$ and $\eta(t):=\|u(t)\|_{\left(H^{1}\right)^{*}}^{2}$ satisfies the problem

$$
\frac{1}{2} \frac{d}{d t} \eta(t)=-\left\langle\partial_{t} u(t), \phi(t)\right\rangle=I_{1}+I_{2}+I_{3},
$$

with $I_{1}, I_{2}$, and $I_{3}$ given in (2.19). The integral $I_{1}$ is estimated in (4.18), whereas for $I_{2}$ and $I_{3}$ we have (2.23). The estimate for $I_{2}$ can be improved in (2.23), using (4.26), Young's inequality for convolutions and Lemma 2.1-(b), as follows:

$$
\begin{aligned}
I_{2} & \leq C \int_{\Omega}\left|D^{2} \mathcal{K} * u_{1}\right||\nabla \phi|^{2} d x \leq C\left\|D^{2} \mathcal{K} * u_{1}\right\|_{L^{\infty}(\Omega)}\|\nabla \phi\|_{L^{2}(\Omega)}^{2} \\
& \leq C\left(\left\|D^{2} \mathcal{K}\right\|_{L^{1}\left(B_{1}(0)\right)}\|u\|_{L^{\infty}(\Omega)}+\left\|D^{2} \mathcal{K}\right\|_{L^{p}\left(\mathbb{R}^{d} \backslash B_{1}(0)\right)}\|u\|_{L^{p /(p-1)}(\Omega)}\right)\|\nabla \phi\|_{L^{2}(\Omega)}^{2} \\
& \leq C \eta(t) .
\end{aligned}
$$

Thus, we get

$$
\frac{d}{d t}\|u(t)\|_{\left(H^{1}\right)^{*}}^{2}+2 \varepsilon\|u(t)\|_{L^{2}}^{2} \leq C\|u(t)\|_{\left(H^{1}\right)^{*}}^{2},
$$

which yields the desired inequality (4.31) by applying Gronwall's inequality.

REMARK 4.4. A crucial point in the proof of Theorem 4.8 is that we need the global Lipschitz continuity of $S_{\varepsilon}(t)$ in the norm of $\left(H^{1}(\Omega)\right)^{*}$. The assumption (4.26) plays an essential role with respect to this issue (see (4.32)). While Newtonian potentials do not satisfy (4.26), in population dynamics the non-local effects are generally modelled with smooth, fast-decaying kernels $\mathcal{K}$ which obey (4.26); see e.g. [53].

The step needed to establish the existence of an exponential attractor is the validity of so-called smoothing property for the difference of two solutions of (4.1)-(4.3). In the present case, such a property is a consequence of the following two lemmas. The first result establishes that the semigroup $S_{\varepsilon}(t)$ is some kind of contraction map, up to the term $\left\|u_{1}-u_{2}\right\|_{L^{2}\left([0, t] ;\left(H^{1}\right)^{*}\right)}$.

Lemma 4.11. Let the assumptions of Proposition 4.10 hold. Then, for every $t \geq 0$, we have:

$$
\left\|u_{1}(t)-u_{2}(t)\right\|_{\left(H^{1}\right)^{*}}^{2} \leq e^{-\kappa t}\left\|u_{1}(0)-u_{2}(0)\right\|_{\left(H^{1}\right)^{*}}^{2}+C_{\varepsilon, M} \int_{0}^{t}\left\|u_{1}(s)-u_{2}(s)\right\|_{\left(H^{1}\right)^{*}}^{2} d s,
$$

for some positive constants $C_{\varepsilon, M}, \kappa$ which depend on $\varepsilon>0, \Omega$, and $\mathcal{K}$. 
Proof. Recall that $u:=u_{1}-u_{2}$. Combining (4.32) together with Poincaré's inequality

$$
\left\|A_{N}^{-1 / 2}(u-\langle u\rangle)\right\|_{L^{2}}^{2}+\langle u\rangle^{2} \leq C_{\Omega}\|u\|_{L^{2}}^{2}
$$

we deduce from (4.32) the following inequality:

$$
\frac{d}{d t}\|u(t)\|_{\left(H^{1}\right)^{*}}^{2}+\frac{2 \varepsilon}{C_{\Omega}}\|u(t)\|_{\left(H^{1}\right)^{*}}^{2} \leq C\|u(t)\|_{\left(H^{1}\right)^{*}}^{2},
$$

for all $t \geq 0$. Thus, Gronwall's inequality entails the desired estimate (4.33).

We now need some compactness for the term $\left\|u_{1}-u_{2}\right\|_{L^{2}\left([0, t] ;\left(H^{1}\right)^{*}\right)}$ on the righthand side of (4.33). This is given by the following lemma.

Lemma 4.12. Let the assumptions of Proposition 4.10 hold. Then, for every $t \geq 0$, the following estimate holds:

$$
\begin{aligned}
& \left\|\partial_{t} u_{1}-\partial_{t} u_{2}\right\|_{L^{2}\left([0, t] ; D\left(A_{N}\right)^{\prime}\right)}^{2}+\varepsilon \int_{0}^{t}\left\|u_{1}(s)-u_{2}(s)\right\|_{L^{2}(\Omega)}^{2} d s \\
\leq & C_{\varepsilon, M} e^{\kappa t}\left\|u_{1}(0)-u_{2}(0)\right\|_{\left(H^{1}\right)^{*}}^{2},
\end{aligned}
$$

where $C_{\varepsilon, M}>0$ and $\kappa>0$ also depend on $\varepsilon, \Omega$, and $\mathcal{K}$.

Proof. The second term on the left-hand side of (4.34) can be easily controlled by (4.31). Thus we only need to estimate the time derivative. Recall that each $\partial_{t} u_{i}$, $i=1,2$, satisfies (4.5). Furthermore, in light of Theorem 4.1, recall that we have

$$
\sup _{t \geq 0}\left\|u_{i}(t)\right\|_{L^{\infty}(\Omega)} \leq C_{\varepsilon, M}, i=1,2 .
$$

Thus, for any test function $w \in D\left(A_{N}\right)$, using the weak formulation (4.5), for $\partial_{t} u:=$ $\partial_{t} u_{1}-\partial_{t} u_{2}$ there holds

$$
\left\langle\partial_{t} u(t), w\right\rangle=I_{4}+I_{5}
$$

where

$$
\begin{aligned}
& I_{4}:=-\left\langle\nabla\left(A_{\varepsilon}\left(u_{1}\right)-A_{\varepsilon}\left(u_{2}\right)\right), \nabla w\right\rangle, \\
& I_{5}:=\left\langle u\left(\nabla \mathcal{K} * u_{1}\right)-u_{2}(\nabla \mathcal{K} * u), \nabla w\right\rangle .
\end{aligned}
$$

First, for every $w \in D\left(A_{N}\right)$ we have

$$
\begin{aligned}
I_{4} & =\left\langle A_{\varepsilon}\left(u_{1}\right)-A_{\varepsilon}\left(u_{2}\right), \Delta w\right\rangle \leq Q\left(\left\|u_{i}\right\|_{L^{\infty}(\Omega)}\right)\left\|u_{1}-u_{2}\right\|_{L^{2}}\|\Delta w\|_{L^{2}} \\
& \leq C\left\|u_{1}-u_{2}\right\|_{L^{2}}\|w\|_{D\left(A_{N}\right)} .
\end{aligned}
$$

On the other hand, it is easy to show, on account of (4.35), that

$$
I_{5} \leq C\left\|u_{1}-u_{2}\right\|_{L^{2}}\|\nabla w\|_{L^{2}} .
$$

These estimates together with (4.31) gives the desired estimate on the time derivative in (4.34).

We now show that the semigroup $S_{\varepsilon}(t)$ is actually uniformly Hölder continuous in the $H^{1-} \cap L^{p}$-norm with respect to the initial data. 
Lemma 4.13. Let $u_{i}(t)=S(t) u_{i}(0)$, with $u_{i}(0) \in \mathcal{Z}_{D N}$. Then, for any $1<p<\infty$, the following estimate is valid:

$$
\left\|u_{1}(t)-u_{2}(t)\right\|_{H^{1-}(\Omega) \cap L^{p}(\Omega)} \leq C_{\varepsilon, M} e^{\kappa t}\left\|u_{1}(0)-u_{2}(0)\right\|_{\left(H^{1}\right)^{*}}^{\gamma},
$$

for all $t \geq t_{*}$, where the constants $C_{\varepsilon, M}>0, \kappa>0$, and $\gamma=\gamma(p)<1$ are independent of the initial data and time.

Proof. Using the interpolation $\left[H^{1},\left(H^{1}\right)^{*}\right]_{1 / 2,2}=L^{2}$, we deduce from estimates (4.31) and (4.6) that

$$
\left\|u_{1}(t)-u_{2}(t)\right\|_{L^{2}(\Omega)} \leq C_{\varepsilon, M} e^{\kappa t}\left\|u_{1}(0)-u_{2}(0)\right\|_{\left(H^{1}\right)^{*}}^{1 / 2},
$$

for all $t \geq t_{*}=t_{+}+1$. By interpolation in the spaces $L^{\infty} \subset L^{p} \subset L^{2}$, for $2<p<\infty, H^{1} \subset$ $H^{1-\delta} \subset\left(H^{1}\right)^{*}, \delta \in(0,1)$, the estimate (4.36) also holds for the difference of solutions $u=u_{1}-u_{2}$.

The last ingredient we need is the uniform Hölder continuity of $t \mapsto S_{\varepsilon}(t) u_{0}$ in the $H^{1-} \cap L^{p}$-norm, which is given by the following result.

Lemma 4.14. Let the assumptions of Proposition 4.10 be satisfied. Consider $u(t)=$ $S_{\varepsilon}(t) u_{0}$ with $u_{0} \in \mathcal{Z}_{D N}$. The following estimate holds:

$$
\|u(t)-u(s)\|_{H^{1-}(\Omega) \cap L^{p}(\Omega)} \leq C_{\varepsilon, M}|t-s|^{\gamma}, \quad \forall t, s \geq t_{*},
$$

where $\gamma=\gamma(p)<1$ and the positive constant $C_{\varepsilon, M}$ is independent of initial data, $u$, and $t$ and $s$.

Proof. According to (4.19) and (4.6), and recalling that $\mathcal{K} \in W_{\text {loc }}^{1,1}\left(\mathbb{R}^{d}\right)$, the following bound holds:

$$
\sup _{t \geq t_{*}}\left\|\Delta A_{\varepsilon} u(t)-\nabla \cdot(u(\nabla \mathcal{K} * u(t)))\right\|_{\left(H^{1}\right)^{*}} \leq C_{\varepsilon, M} .
$$

Consequently, by comparison in (4.5), we have that

$$
\sup _{t \geq t_{*}}\left\|\partial_{t} u(t)\right\|_{\left(H^{1}\right)^{*}} \leq C_{\varepsilon, M}
$$

which entails

$$
\|u(t)-u(s)\|_{\left(H^{1}\right)^{*}} \leq C_{\varepsilon, M}|t-s|, \quad \forall t, s \geq t_{*} .
$$

Estimate (4.38) now follows from (4.39) and standard interpolation inequalities, as in the proof of Lemma 4.13 .

Proof of Theorem 4.8. In order to apply Proposition 4.9, it is sufficient to verify the existence of an exponential attractor for the restriction of $S(t)$ on some properly chosen semi-invariant absorbing set in $\mathcal{Z}_{D N}$. Recall that, by (4.19) and Lemma 4.2 , the ball $B_{0}:=B_{C^{\alpha}(\bar{\Omega}) \cap H^{1}(\Omega)}\left(C_{\varepsilon, M}\right)$ will be absorbing for $S_{\varepsilon}(t)$ provided that $C_{\varepsilon, M}>0$ is sufficiently large. Since we want this ball to be semi-invariant with respect to the semigroup, we push it forward by the semigroup, by defining first the set $B_{1}=\left[\cup_{t \geq 0} S_{\varepsilon}(t) B_{0}\right]_{\left(H^{1}\right)^{*}}$, where $[\cdot]_{\left(H^{1}\right)^{*}}$ denotes closure in the space $\left(H^{1}(\Omega)\right)^{*}$. Then set $\mathbb{B}=S(1) B_{1}$. Thus, $\mathbb{B}$ is a semi-invariant compact (for the metric of $\left(H^{1}(\Omega)\right)^{*}$ ) 
subset of the phase space $\mathcal{Z}_{D N}$. On the other hand, due to the results proven in this section, we have

$$
\sup _{t \geq 0}\left(\|u(t)\|_{C^{\alpha}(\bar{\Omega}) \cap H^{1}(\Omega)}+\left\|A_{\varepsilon} u(t)\right\|_{H^{1}(\Omega)}+\left\|\partial_{t} u(t)\right\|_{\left(H^{1}\right)^{*}}\right) \leq C_{\varepsilon, M},
$$

for every trajectory $u$ originating from $u_{0} \in \mathbb{B}$, for some positive constant $C_{\varepsilon, M}$ which is independent of the choice of $u_{0} \in \mathbb{B}$. We can now apply the abstract result above to the map $\mathbb{S}=S_{\varepsilon}(T)$ and $\mathcal{H}=\left(H^{1}(\Omega)\right)^{*}$, for a fixed $T>0$ such that $e^{-\kappa T}<\frac{1}{2}$, where $\kappa>0$ is the same as in Lemma 4.11. To this end, we introduce the functional spaces

$$
\mathcal{V}_{1}:=L^{2}\left([0, T] ; L^{2}(\Omega)\right) \cap H^{1}\left([0, T] ; D\left(A_{N}\right)^{\prime}\right), \quad \mathcal{V}:=L^{2}\left([0, T] ;\left(H^{1}(\Omega)\right)^{*}\right),
$$

and note that $\mathcal{V}_{1}$ is compactly embedded into $\mathcal{V}$. Finally, we introduce the operator $\mathbb{T}$ : $\mathbb{B} \rightarrow \mathcal{V}_{1}$ by $\mathbb{T} u_{0}:=u \in \mathcal{V}_{1}$, where $u$ solves (4.1)-(4.3) with $u(0)=u_{0} \in \mathbb{B}$. We claim that the maps $\mathbb{S}$, $\mathbb{T}$, and the spaces $\mathcal{H}, \mathcal{V}$, and $\mathcal{V}_{1}$ thus defined satisfy all the assumptions of Proposition 4.9. Indeed, the global Lipschitz continuity (4.29) of $\mathbb{T}$ is an immediate corollary of Lemma 4.12, and estimate (4.30) follows from estimate (4.33). Therefore, due to Proposition 4.9, the semigroup $\mathbb{S}(n)=S_{\varepsilon}(n T)$ generated by the iterations of the operator $\mathbb{S}: \mathbb{B} \rightarrow \mathbb{B}$ possesses a (discrete) exponential attractor $\mathcal{M}_{d}$ in $\mathbb{B}$ endowed by the topology of $\left(H^{1}(\Omega)\right)^{*}$. In order to construct the exponential attractor $\mathcal{E}$ for the semigroup $S_{\varepsilon}(t)$ with continuous time, we note that, due to Lemma 4.10, this semigroup is Lipschitz continuous with respect to the initial data in the topology of $\left(H^{1}(\Omega)\right)^{*}$. Moreover, by (4.36) and (4.38) the map $\left(t, u_{0}\right) \mapsto S_{\varepsilon}(t) u_{0}$ is also uniformly Hölder continuous on $[0, T] \times \mathbb{B}$, where $\mathbb{B}$ is endowed with the metric topology of $\left(H^{1}(\Omega)\right)^{*}$. Hence, the desired exponential attractor $\mathcal{M}$ for the continuous semigroup $S_{\varepsilon}(t)$ can be obtained by the standard formula

$$
\mathcal{M}=\bigcup_{t \in[0, T]} S_{\varepsilon}(t) \mathcal{M}_{d}
$$

In order to finish the proof of the theorem, we only need to verify that $\mathcal{M}$ defined as above will be the exponential attractor for $S_{\varepsilon}(t)$ restricted to $\mathbb{B}$ not only with respect to the $\left(H^{1}(\Omega)\right)^{*}$-metric, but also in with respect to a stronger metric. This is an immediate corollary of the fact that $\mathbb{B}$ is bounded in $C^{\alpha}(\bar{\Omega}) \cap H^{1}(\Omega)$, along with standard interpolation inequalities between the following spaces: $L^{\infty} \subset L^{p} \subset L^{2}$, $H^{1} \subset H^{1-\delta} \subset\left(H^{1}\right)^{*}$, for $2<p<\infty$ and $\delta \in(0,1]$. Theorem 4.8 is now proved.

\section{Convergence to steady states}

In this section, we show that any global-in-time bounded solution to the model (4.1)-(4.3) converges to a single equilibrium of (4.21) as time tends to infinity. The proof of the main result is based on a suitable version of the Eojasiewicz-Simon theorem and propositions 4.4 and 4.5. The question of such convergence is usually a delicate matter since it is well known that the topology of the set of stationary solutions of (4.21) can be non-trivial. In particular, there may be a continuum of stationary solutions for (4.21) even in the simplest cases; for instance when $\Omega$ is a disk, $\mathcal{K}$ is either a Newtonian or Bessel potential and $A(y) \equiv y$ (see $[39,50])$.

The main result of this section reads as follows.

TheOrem 5.1. Let the assumptions of Theorem 4.1 be satisfied. Assume that $\Phi$ is a real analytic function on $\mathbb{R}_{+}$. For any $u_{0} \in \mathcal{Z}_{D N}$ with $\left\langle u_{0}\right\rangle>0$, the corresponding 
positive solution $u(t)=S_{\varepsilon}(t) u_{0}$ to the non-degenerate aggregation equations (4.1)(4.3) converges to a single stationary state $u_{*}$ of (4.21) in the sense that

$$
\lim _{t \rightarrow \infty}\left\|u(t)-u_{*}\right\|_{L^{p}(\Omega)}=0,
$$

for any $p>1$. Moreover, there exist constants $C>0, \rho=\rho\left(p, u_{*}\right) \in(0,1)$ such that the following convergence rate holds:

$$
\left\|u(t)-u_{*}\right\|_{L^{p}(\Omega)} \leq C(1+t)^{-\rho}
$$

for all $t \geq 0$.

Owing to (4.20), Proposition 4.4-(iii), Proposition 4.5, and the regularity properties of $u \in C^{\alpha / 2}\left((0 ; \infty) ; C^{\alpha}(\bar{\Omega})\right)$, we may then assume without loss of generality that

$$
\inf _{x \in \Omega} u(t, x) \geq \underline{u}>0, \text { for all } t>0 .
$$

We employ a generalized version of the Eojasiewicz-Simon theorem proved in [31, Theorem 6] (cf. also [28, Lemma 2.20]). The version that applies to our case is formulated in the following.

Lemma 5.2. Under the assumptions of Theorem 5.1, there exist constants $\theta \in\left(0, \frac{1}{2}\right]$, $C>0$, and $\delta>0$ such that the following inequality holds:

$$
\left|\mathcal{E}_{\varepsilon}(u)-\mathcal{E}_{\varepsilon}\left(u_{*}\right)\right|^{1-\theta} \leq C\|\mu-\langle\mu\rangle\|_{L^{2}(\Omega)},
$$

for all $u \in L^{\infty}(\Omega) \cap H^{1}(\Omega)$ provided that $\left\|u-u_{*}\right\|_{L^{2}(\Omega)} \leq \delta$. Here $\mu=\mu(u)$ denotes $\Phi_{\varepsilon}^{\prime}(u)-\mathcal{K} * u$.

Proof. We will now apply the abstract result [31, Theorem 6] to the energy functional $\mathcal{E}_{\varepsilon}(u)$, which according to $(4.22)$ is the sum of entropy and an interface energy term. In contrast to this feature, we shall split $\mathcal{E}_{\varepsilon}(\varphi)$ into the sum of a convex (entropy) functional $\Sigma: L^{2}(\Omega) \rightarrow \mathbb{R} \cup\{\infty\}$, with a suitable effective domain, and a non-local interaction functional $\Psi: L^{2}(\Omega) \rightarrow \mathbb{R}$. Let $\underline{u}>0$ be the lower bound from (5.3) and recall that $\varepsilon>0$. We define the lower-semicontinuous and strongly convex functional $\Sigma=\Sigma_{\varepsilon}$ by

$$
\Sigma(u):= \begin{cases}\int_{\Omega}\left(\Phi_{\varepsilon}(u)-\varepsilon \ln (\underline{u}) u\right) d x, & \text { if } u \in L^{\infty}(\Omega), u \geq 0, \\ +\infty, & \text { otherwise, }\end{cases}
$$

with closed effective domain $\operatorname{dom}(\Sigma)=\mathcal{Z}_{D N} \cap H^{1}(\Omega)$, and the quadratic functional $\Psi=\Psi_{\varepsilon}: L^{2}(\Omega) \rightarrow \mathbb{R}$, given by

$$
\Psi(u):=-\frac{1}{2} \int_{\Omega}[u(\mathcal{K} * u)-2 \varepsilon \ln (\underline{u}) u] d x .
$$

We have that $\Sigma$ is Fréchet differentiable on any open subset $\bar{U}$ of

$$
U_{M}:=\left\{\psi \in L^{\infty}(\Omega):|\langle\psi\rangle| \leq M ; \underline{u} \leq \psi(x) \leq C_{M}\right\},
$$

(where $C_{M}>0$ is such that $\|\psi\|_{L^{\infty}(\Omega)} \leq C_{M}$, since $\psi$ is bounded) with Fréchet derivative $D \Sigma: \bar{U} \rightarrow L^{\infty}(\Omega)$ having the form

$$
\langle D \Sigma(u), \xi\rangle=\int_{\Omega}\left(\Phi_{\varepsilon}^{\prime}(u)-\varepsilon \ln (\underline{u})\right) \cdot \xi d x
$$


for all $u \in \bar{U}$ and $\xi \in L^{\infty}(\Omega)$. The analyticity of $D \Sigma$ as a mapping on $L^{\infty}(\Omega)$ is standard owing to the analyticity of $\Phi$ (see, e.g., [31, Remark 3]). Moreover, due to assumptions on $A$ in Theorem 4.1, we have $\Phi^{\prime}(y) \sim A^{\prime}(y) \sim y^{m-1}$, and recalling that $\Phi_{\varepsilon}^{\prime}(y)=\Phi^{\prime}(y)+\varepsilon \ln (y)$, one has $\Phi^{\prime}(\underline{u})>0$ and

$$
\left\langle D \Sigma\left(u_{1}\right)-D \Sigma\left(u_{2}\right), u_{1}-u_{2}\right\rangle \geq \Phi^{\prime}(\underline{u})\left\|u_{1}-u_{2}\right\|_{L^{2}(\Omega)}^{2},
$$

for all $u_{1}, u_{2} \in \bar{U}$, and

$$
\left\|D \Sigma\left(u_{1}\right)-D \Sigma\left(u_{2}\right)\right\|_{\left(L^{2}(\Omega)\right)^{*}} \leq \gamma\left\|u_{1}-u_{2}\right\|_{L^{2}(\Omega)},
$$

for some positive constant $\gamma=\gamma\left(C_{M}, \underline{u}, \varepsilon\right)$. Moreover, computing the second Fréchet derivative $D^{2} \Sigma$ of $\Sigma$,

$$
\left\langle D^{2} \Sigma(u) \xi_{1}, \xi_{2}\right\rangle=\int_{\Omega} \Phi_{\varepsilon}^{\prime \prime}(u) \xi_{1} \cdot \xi_{2} d x,
$$

yields that $D^{2} \Sigma \in \mathcal{L}\left(L^{\infty}(\Omega), L^{\infty}(\Omega)\right)$ is an isomorphism for every $u \in \bar{U}$, owing to the fact that $\Phi_{\varepsilon}^{\prime \prime}(u)=A_{\varepsilon}^{\prime}(u) / u \geq \zeta=\zeta\left(C_{M}, \underline{u}\right)>0$. Concerning the (quadratic) function $\Psi$, we see that

$$
\Psi(u)=\frac{1}{2}\langle-\mathcal{K} * u, u\rangle_{L^{2}(\Omega)}+\langle\varepsilon \ln (\underline{u}), u\rangle_{L^{2}(\Omega)}, \forall u \in L^{2}(\Omega) .
$$

We recall that the linear operator $\psi \mapsto \mathcal{K} * \psi$ is self-adjoint and compact from $L^{2}(\Omega)$ to itself and is also compact from $L^{\infty}(\Omega)$ to $C^{0}(\bar{\Omega})$ (since $\mathcal{K} \in W_{\text {loc }}^{1,1}$ ). On the other hand, we also have the following (orthogonal) sum decomposition of $L^{2}(\Omega)=L_{0}^{2}(\Omega) \oplus H_{1}$, where

$$
L_{0}^{2}(\Omega):=\left\{u \in L^{2}(\Omega):\langle u\rangle=0\right\}, H_{1}:=\left\{u \in L^{2}(\Omega): u=\text { const. }\right\}
$$

Then, the annihilator of $L_{0}^{2}(\Omega)$ is the one-dimensional subspace

$$
L_{0}^{0}:=\left\{\operatorname{ch} \in\left(L^{2}(\Omega)\right)^{*}: c \in \mathbb{R}\right\}
$$

of constant functions, where $h \in\left(L^{2}(\Omega)\right)^{*} \simeq L^{2}(\Omega)$ is given by $\langle h, u\rangle=\frac{1}{|\Omega|} \int_{\Omega} u d x, u \in$ $L^{2}(\Omega)$. Hence, the hypotheses of [31, Theorem 6] are satisfied and the sum

$$
\mathcal{E}_{\varepsilon}=\Sigma+\Psi: L^{2}(\Omega) \rightarrow \mathbb{R} \cup\{\infty\}
$$

is a well defined, bounded from below functional with nonempty, closed, and convex effective domain $\operatorname{dom}\left(\mathcal{E}_{\varepsilon}\right)=\operatorname{dom}(\Sigma)$. Unravelling notation in [31, Theorem 6], and observing that the Fréchet derivative

$$
D \mathcal{E}_{\varepsilon}(u)=\Phi_{\varepsilon}^{\prime}(u)-\mathcal{K} * u=: \mu,
$$

we have

$$
\begin{aligned}
\left|\mathcal{E}_{\varepsilon}(u)-\mathcal{E}_{\varepsilon}\left(u_{*}\right)\right|^{1-\theta} & \leq C \inf _{u \in L^{2}(\Omega)}\left\{\left\|D \mathcal{E}_{\varepsilon}(u)-\mu_{*}\right\|_{L^{2}(\Omega)}: \mu_{*} \in L_{0}^{0}\right\} \\
& =C\|\mu-\langle\mu\rangle\|_{L^{2}(\Omega)}
\end{aligned}
$$

from which (5.4) follows. 
We are now in a position to complete the proof of Theorem 5.1.

Proof. [Proof of Theorem 5.1.] We argue as in the proof of [28, Theorem 2.21]. First, we note that by virtue of the regularity results proven in the previous section (see, e.g., (4.40)), all $u_{*} \in \omega\left[u_{0}\right]$ are bounded in $C^{\alpha}(\bar{\Omega}) \cap H^{1}(\Omega)$. Besides, recalling Proposition 4.4-(ii), we have

$$
\mathcal{E}_{\varepsilon}(u(t)) \rightarrow \mathcal{E}_{\varepsilon, \infty}, \text { as } t \rightarrow \infty,
$$

and the limit energy $\mathcal{E}_{\varepsilon, \infty}$ is the same for every steady-state solution $u_{*} \in \omega\left[u_{0}\right]$. Moreover, we can integrate $(4.23)$ over $(t, \infty)$ to get

$$
\begin{aligned}
& \underline{u} \int_{t}^{\infty} \int_{\Omega}\left|\nabla \Phi_{\varepsilon}^{\prime}(u(t))-\nabla \mathcal{K} * u(t)\right|^{2} d x d s \\
\leq & \int_{t}^{\infty} \int_{\Omega} u(t)\left|\nabla \Phi_{\varepsilon}^{\prime}(u(t))-\nabla \mathcal{K} * u(t)\right|^{2} d x d s \\
= & \mathcal{E}_{\varepsilon}(u(t))-\mathcal{E}_{\varepsilon, \infty}=\mathcal{E}_{\varepsilon}(u(t))-\mathcal{E}_{\varepsilon}\left(u_{*}\right) .
\end{aligned}
$$

By virtue of Lemma 5.2 and recalling that $\mu(t)=\Phi_{\varepsilon}^{\prime}(u(t))-\nabla \mathcal{K} * u(t)$, we have

$$
\left|\mathcal{E}_{\varepsilon}(u(t))-\mathcal{E}_{\varepsilon}\left(u_{*}\right)\right|^{1-\theta} \leq C\|\mu(t)-\langle\mu(t)\rangle\|_{L^{2}(\Omega)} \leq C\|\nabla \mu(t)\|_{L^{2}(\Omega)}
$$

exploiting Poincaré's inequality, provided that

$$
\left\|u-u_{*}\right\|_{L^{2}(\Omega)} \leq \delta .
$$

This, combined with the previous identity, yields

$$
\int_{t}^{\infty}\|\nabla \mu(s)\|_{L^{2}(\Omega)}^{2} d s \leq C\|\nabla \mu(t)\|_{L^{2}(\Omega)}^{\frac{1}{(1-\theta)}},
$$

for all $t>0$, for as long as (5.7) holds. Note that, in general, the quantities $\theta, C$, and $\delta$ above may depend on $u_{*}>0$ and $\varepsilon>0$. Finally, let us set

$$
\hat{W}=\cup\{\mathcal{I}: \mathcal{I} \text { is an open interval on which (5.7) holds }\} .
$$

Clearly, $\hat{W}$ is nonempty since $u_{*} \in \omega\left[u_{0}\right]$. We can now use (5.8), the fact that $\|\nabla \mu(t)\|_{L^{2}(\Omega)} \in L^{2}(0, \infty)$, and exploit [26, Lemma 5.1] (with $\alpha=2(1-\theta)$ ) to deduce that $\|\nabla \mu(\cdot)\|_{L^{2}(\Omega)} \in L^{1}(\hat{W})$ and

$$
\int_{\hat{W}}\|\nabla \mu(s)\|_{L^{2}(\Omega)} d s \leq C\left(u_{*}, \underline{u}\right)<\infty .
$$

Consequently, using the bound (5.9) and the main equation (4.1), which also reads $\partial_{t} u(t)=\operatorname{div}(u(t) \nabla \mu(t))$, we obtain

$$
\int_{\hat{W}}\left\|\partial_{t} u(s)\right\|_{\left(H^{1}(\Omega)\right)^{*}} d s \leq C<\infty .
$$

In order to finish the proof of the convergence result in (5.1) it suffices to show that it holds in $L^{2}$-norm. Indeed, in this case (5.1) will become an immediate consequence of the $L^{2}-\left(C^{\alpha} \cap H^{1}\right)$ smoothing property of the bounded solutions $u(t)$ and all $u_{*} \in$ $\omega\left[u_{0}\right]$. We claim that we can find a sufficiently large time $\tau>0$ such that $(\tau, \infty) \subset$ 
$\hat{W}$. To this end, recalling (5.5) and the above bounds, we also have that $\partial_{t} \varphi \in$ $L^{2}\left(0, \infty ;\left(H^{1}(\Omega)\right)^{*}\right), \nabla \mu \in L^{2}\left(0, \infty ;\left(L^{2}(\Omega)\right)^{d}\right)$ and, furthermore, for any $k>0$ there exists a time $t_{*}=t_{*}(k)>0$ such that

$$
\left\|\partial_{t} u\right\|_{L^{1}\left(\hat{W} \cap\left(t_{*}, \infty\right) ;\left(H^{1}\right)^{*}\right)} \leq k,\left\|\partial_{t} u\right\|_{L^{2}\left(\left(t_{*}, \infty\right) ;\left(H^{1}\right)^{*}\right)} \leq k,\|\nabla \mu\|_{L^{2}\left(\left(t_{*}, \infty\right) ;\left(L^{2}\right)^{d}\right)} \leq k .
$$

Next, observe that by the regularity properties of $u$ (see Section 3), there is a time $t_{\#}>0$ such that

$$
\sup _{t \geq t_{\#}}\|u(t)\|_{H^{1} \cap C^{\alpha}(\bar{\Omega})} \leq C .
$$

Now, let $\left(t_{0}, t_{2}\right) \subset \hat{W}$, for some $t_{2}>t_{0} \geq t_{*}(k),\left|t_{0}-t_{2}\right| \geq 1$ such that (5.12) holds (without loss of generality, we can assume that $\left.t_{*} \geq t_{\#}\right)$. This claim is an immediate consequence of the aforementioned $L^{2}-\left(H^{1} \cap C^{\alpha}(\bar{\Omega})\right)$ smoothing property and bounds (5.11). Using (5.11) and (5.12), we obtain

$$
\begin{aligned}
\left\|u\left(t_{0}\right)-u\left(t_{2}\right)\right\|_{L^{2}(\Omega)}^{2} & =2 \int_{t_{0}}^{t_{2}}\left\langle\partial_{t} u(s), u(s)-u\left(t_{0}\right)\right\rangle_{L^{2}(\Omega)} d s \\
& \leq 2 \int_{t_{0}}^{t_{2}}\left\|\partial_{t} u(s)\right\|_{\left(H^{1}(\Omega)\right)^{*}}\left(\|u(s)\|_{H^{1}(\Omega)}+\left\|u\left(t_{0}\right)\right\|_{H^{1}(\Omega)}\right) d s \\
& \leq C\left\|\partial_{t} u\right\|_{L^{1}\left(t_{0}, t_{2} ;\left(H^{1}\right)^{*}\right)}\left(\|u\|_{L^{\infty}\left(t_{*}, \infty ; H^{1}\right)}+1\right) \leq C k
\end{aligned}
$$

Therefore we can choose a time $t_{*}(k)=\tau<t_{0}<t_{2}$, such that

$$
\left\|u\left(t_{0}\right)-u\left(t_{2}\right)\right\|_{L^{2}(\Omega)}<\frac{\delta}{3}
$$

provided that (5.7) holds for all $t \in\left(t_{0}, t_{2}\right)$. Since $u_{*} \in \omega\left[u_{0}\right]$, a large (redefined) $\tau$ can be chosen such that

$$
\left\|u(\tau)-u_{*}\right\|_{L^{2}(\Omega)}<\frac{\delta}{3}
$$

whence (5.14) yields $(\tau, \infty) \subset \hat{W}$. Indeed, taking

$$
\bar{t}=\inf \left\{t>\tau:\left\|u(t)-u_{*}\right\|_{L^{2}(\Omega)} \geq \delta\right\},
$$

we have $\bar{t}>\tau$ and $\left\|u(\bar{t})-u_{*}\right\|_{L^{2}(\Omega)} \geq \varepsilon$ if $\bar{t}$ is finite. On the other hand, in view of (5.14) and (5.15), we have

$$
\left\|u(t)-u_{*}\right\|_{L^{2}(\Omega)} \leq\|u(t)-u(\tau)\|_{L^{2}(\Omega)}+\left\|u(\tau)-u_{*}\right\|_{L^{2}(\Omega)}<\frac{2 \delta}{3},
$$

for all $\bar{t}>t \geq \tau$, and this leads to a contradiction. Therefore, $\bar{t}=\infty$ and by (5.11) the integrability of $\partial_{t} u$ in $L^{1}\left(\tau, \infty ;\left(H^{1}(\Omega)\right)^{*}\right)$ follows. Hence, $\omega\left[u_{0}\right]=\left\{u_{*}\right\}$ and (5.1) holds on account of the $L^{2}-\left(H^{1} \cap C^{\alpha}(\bar{\Omega})\right)$ smoothing property. The proof is finished.

Proof. [Proof of (5.2).] Without loss of generality, suppose now that, for all $t \geq t_{*}>0$, we have $\mathcal{E}_{\varepsilon}(u(t))>\mathcal{E}_{\varepsilon}\left(u_{*}\right)$ (otherwise, there is nothing to prove). Define the function

$$
\Xi(t):=\mathcal{E}_{\varepsilon}(u(t))-\mathcal{E}_{\varepsilon}\left(u_{*}\right)
$$


and observe that by (5.5)-(5.6) and (4.23), it satisfies

$$
\frac{d}{d t} \Xi(t)+C \Xi(t)^{2(1-\theta)} \leq 0, \text { for all } t \geq t_{*}
$$

for some positive constant $C=C(\underline{u})$. Integration of the preceding inequality yields

$$
\Xi(t) \leq \Xi(0)\left(1+C \Xi(0)^{1-2 \theta} t\right)^{-\frac{1}{1-2 \theta}},
$$

for all $t \geq t_{*}$. On the other hand, we have by (5.5) that

$$
-\frac{d}{d t} \Xi(t)^{\theta}=-\theta \Xi(t)^{\theta-1} \frac{d}{d t} \Xi(t) \geq C \theta\|\nabla \mu(t)\|_{L^{2}(\Omega)},
$$

for all $t \geq t_{*}$, provided that $\left\|u(t)-u_{*}\right\| \leq \delta$. Integrating this inequality over $(t, \infty)$, we also get

$$
\int_{t}^{\infty}\|\nabla \mu(s)\|_{L^{2}(\Omega)} d s \leq C<\infty
$$

for all $t \geq t_{*}$. As above, we obtain

$$
\int_{t}^{\infty}\left\|\partial_{t} u(s)\right\|_{\left(H^{1}(\Omega)\right)^{*}} d s \leq C<\infty
$$

and combining with (5.5) and (5.16) yields

$$
\begin{aligned}
\left\|u(t)-u_{*}\right\|_{\left(H^{1}(\Omega)\right)^{*}} & \leq \int_{t}^{\infty}\left\|\partial_{t} u(s)\right\|_{\left(H^{1}(\Omega)\right)^{*}} d s \leq C \int_{t}^{\infty}\|\nabla \mu(s)\|_{L^{2}(\Omega)} d s \\
& \leq C(1+t)^{-\frac{\theta}{1-2 \theta}}
\end{aligned}
$$

for some positive constant $C$, which depends on $\Xi(0), \theta, \underline{u}$, and $\varepsilon$. By using standard interpolation inequalities (see, Section 4 ) one can deduce the convergence rate estimate in the stronger norm in (5.2). The proof is complete.

Acknowledgment. The author thanks the anonymous referees for their careful reading of the manuscript and many useful comments.

\section{REFERENCES}

[1] M. Aida and A. Yagi, Global stability of approximation for exponential attractors, Funkcial. Ekvac., 47, 251-276, 2004.

[2] M. Aida and A. Yagi, Global attractor for approximate system of chemotaxis and growth, Second International Conference on Dynamics of Continuous, Discrete and Impulsive Systems (London, ON, 2001), Dyn. Contin. Discrete Impuls. Syst. Ser. A Math. Anal., 10, 309-315, 2003.

[3] M. Aida, T. Tsujikawa, M. Efendiev, A. Yagi, and M. Mimura, Lower estimate of the attractor dimension for a chemotaxis growth system, J. London Math. Soc., 74, 453-474, 2006.

[4] J. Blat and K.J. Brown, Bifurcation of steady-state solutions in predator-prey and competition systems, Proc. Roy. Soc. Edinburgh Sect. A, 97, 21-34, 1984.

[5] J. Bedrossian, Global minimizers for free energies of subcritical aggregation equations with degenerate diffusion, Appl. Math. Lett., 24, 1927-1932, 2011.

[6] P. Biler and L. Brandolese, On the parabolic-elliptic limit of the doubly parabolic Keller-Segel system modelling chemotaxis, Studia Math., 193, 241-261, 2009.

[7] M. Burger, V. Capasso, and D. Morale, On an aggregation model with long and short range interactions, Nonlinear Anal. Real World Appl., 8, 939-958, 2007. 
[8] M. Burger and M. Di Francesco, Large time behavior of nonlocal aggregation models with nonlinear diffusion, Netw. Heterog. Media, 3, 749-785, 2008.

[9] M. Burger, M. Di Francesco, and M. Franek, Stationary states of quadratic diffusion equations with long-range attraction, arXiv:1103.5365, Commun. Math. Sci., 11, 709-739, 2013.

[10] P.W. Bates and J. Han, The Neumann boundary problem for a nonlocal Cahn-Hilliard equation, J. Diff. Equ., 212, 235-277, 2005.

[11] P.W. Bates and J. Han, The Dirichlet boundary problem for a nonlocal Cahn-Hilliard equation, J. Math. Anal. Appl., 311, 289-312, 2005.

[12] A.L. Bertozzi and D. Slepčev, Existence and uniqueness of solutions to an aggregation equation with degenerate diffusion, Comm. Pure Appl. Anal., 9(6), 1617-1637, 2010.

[13] A.L. Bertozzi and J. Brandman, Finite-time blow-up of $L^{\infty}$-weak solutions of an aggregation equation, Comm. Math. Sci., 8, 45-65, 2010.

[14] A.L. Bertozzi, J.A. Carrillo, and T. Laurent, Blowup in multidimensional aggregation equations with mildly singular interaction kernels, Nonlin., 22, 683-710, 2009.

[15] A.L. Bertozzi and T. Laurent, Finite-time blow-up of solutions of an aggregation equation in $R^{n}$, Comm. Math. Phys., 274, 717-735, 2007.

[16] A.L. Bertozzi, T. Laurent, and J. Rosado, $L^{p}$ theory for the multidimensional aggregation equation, Comm. Pure Appl. Math., 64(1), 45-83, 2011.

[17] J. Bedrossian, N. Rodríguez, and A.L. Bertozzi, Local and global well-posedness for aggregation equations and Patlak-Keller-Segel models with degenerate diffusion, Nonlin., 24(6), 1683$1714,2011$.

[18] V.V. Chepyzhov, M.I. Vishik, and S.V. Zelik, Strong trajectory attractors for dissipative Euler equations, J. Math. Pures Appl., 96, 395-407, 2011.

[19] Y. Chen, Hölder estimates for solutions of uniformly degenerate quasilinear parabolic equations, Chinese Ann. Math. Ser. B, 5, 661-678, 1984.

[20] L. Dung, Remarks on Hölder continuity for parabolic equations and convergence to global attractors, Nonlin. Anal., 41, 921-941, 2000.

[21] E. Di Benedetto and A. Friedman, Hölder estimates for nonlinear degenerate parabolic systems, J. Reine Angew. Math., 357, 1-22, 1985.

[22] G. Dong, Nonlinear Partial Differential Equations of Second Order, Transi. Math. Monographs, Amer. Math. Soc., Providence, RI, 1991.

[23] L.C. Evans, Partial Differential Equations, Second Edition, Graduate Studies in Mathematics 19, American Mathematical Society, Providence, RI, 2010.

[24] M. Efendiev and S. Zelik, Finite-dimensional attractors and exponential attractors for degenerate doubly nonlinear equations, Math. Meth. Appl. Sci., 32, 1638-1668, 2009.

[25] M. Efendiev, E. Nakaguchi, and K. Osaki, Dimension estimate of the exponential attractor for the chemotaxis-growth system, Glasg. Math. J., 50(3), 483-497, 2008.

[26] E. Feireisl, P. Laurençot, and H. Petzeltová, On convergence to equilibria for the Keller-Segel chemotaxis model, J. Diff. Equ., 236, 551-569, 2007.

[27] C.G. Gal, On a class of degenerate parabolic equations with dynamic boundary conditions, J. Diff. Equ., 253, 126-166, 2012.

[28] C.G. Gal and M. Grasselli, Longtime behavior of nonlocal Cahn-Hilliard equations, eprint arXiv:1207.4018, Disc. Cont. Dyn. Sys. (Series A), 34, 145-179, 2014.

[29] G. Giacomin and J.L. Lebowitz, Phase segregation dynamics in particle systems with long range interactions. I. Macroscopic limits, J. Statist. Phys., 87, 37-61, 1997.

[30] G. Giacomin and J.L. Lebowitz, Phase segregation dynamics in particle systems with long range interactions. II. Phase motion, SIAM J. Appl. Math., 58, 1707-1729, 1998.

[31] H. Gajewski and J.A. Griepentrog, A descent method for the free energy of multicomponent systems, Disc. Cont. Dyn. Sys., 15, 505-528, 2006.

[32] A.V. Ivanov, Quasilinear degenerate and nonuniformly elliptic and parabolic equations of second order, Trudy Mat. Inst. Steklov., 160, 1-285, 1982; English transi., Proc. Steklov Inst. Math., 160, 1-287, 1984.

[33] N.V. Krylov, Nonlinear Elliptic and Parabolic Equations of Second Order, "Nauka", Moscow, 1985; English transi., Reidel, Dodrecht, 1987.

[34] E.F. Keller and L.A. Segel, Initiation of slime mold aggregation viewed as an instability, J. Theo. Biol., 26, 399-415, 1970.

[35] E.F. Keller and L.A. Segel, Model for chemotaxis, J. Theo. Biol., 30, 225-234, 1971.

[36] E.F. Keller and L.A. Segel, Traveling bands of chemotactic bacteria: A theoretical analysis, J. Theo. Biol., 30, 235-248, 1971.

[37] R. Kowalczyk, Preventing blow-up in a chemotaxis model, J. Math. Anal. Appl., 305, 566-588, 2005.

[38] J. Han, The Cauchy problem and steady state solutions for a nonlocal Cahn-Hilliard equation, 
Electron. J. Diff. Equ., 113, 9, 2004.

[39] G. Harada, T. Nagai, T. Senba, and T. Suzuki, Concentration lemma, Brezis-Merle type inequality, and a parabolic system of chemotaxis, Adv. Diff. Equ., 6, 1255-1280, 2001.

[40] D. Horstmann, From 1970 until present: The Keller-Segel model in chemotaxis and its consequences, part 1, Jahresber. Deutsch. Math.-Verein., 105, 103-165, 2003.

[41] D. Horstmann, From 1970 until present: The Keller-Segel model in chemotaxis and its consequences, part 2, Jahresber. Deutsch. Math.-Verein., 106, 51-69, 2004.

[42] D. Hilhorst, S. Martin, and M. Mimura, Singular limit of a competition-diffusion system with large interspecific interaction, J. Math. Anal. Appl., 390, 488-513, 2012.

[43] S.O. Londen and H. Petzeltová, Convergence of solutions of a non-local phase-field system, Disc. Cont. Dyn. Sys. Ser. S, 4(3), 653-670, 2011.

[44] T. Namba and M. Mimura, Spatial distribution of competing populations, J. Theo. Biol., 87, 795-814, 1980.

[45] A. Miranville and S. Zelik, Attractors for dissipative partial differential equations in bounded and unbounded domains, Handbook of Differential Equations: Evolutionary Equations, Handb. Diff. Equ., Elsevier/North-Holland, Amsterdam, 103-200, 2008.

[46] V. Pata and S. Zelik, A result on the existence of global attractors for semigroups of closed operators, Comm. Pure Appl. Anal., 6(2), 481-486, 2007.

[47] M.M. Porzio and V. Vespri, Holder estimates for local solutions of some doubly nonlinear degenerate parabolic equations, J. Diff. Equ., 103, 146-178, 1993.

[48] J.C. Robinson, Infinite-Dimensional Dynamical Systems. An Introduction to Dissipative Parabolic PDEs and the Theory of Global Attractors, Cambridge Texts in Applied Mathematics, Cambridge University Press, Cambridge, 2001.

[49] A. Schiaffino and A. Tesei, Competition systems with Dirichlet boundary conditions, J. Math. Biol., 15, 93-105, 1982.

[50] T. Senba and T. Suzuki, Some structures of the solution set for a stationary system of chemotaxis, Adv. Math. Sci. Appl., 10, 191-224, 2000.

[51] R. Temam, Infinite-Dimensional Dynamical Systems in Mechanics and Physics, SpringerVerlag, New York, 1997.

[52] Y. Takei, T. Tsujikawa, and A. Yagi, Numerical computations and pattern formation for absobate-induced phase transition model, Scientiae Mathematicae Japonicae Online, electronic, 55-64, 2005.

[53] C.M. Topaz, A.L. Bertozzi, and M.A. Lewis, A nonlocal continuum model for biological aggregation, Bull. Math. Biol., 68(7), 1601-1623, 2006.

[54] Y. Zhang and S. Zheng, Asymptotic behavior of solutions to a quasilinear nonuniform parabolic system modelling chemotaxis, J. Diff. Equ., 248, 1684-1710, 2010. 